\title{
Revisiting big-bang nucleosynthesis constraints on long-lived decaying particles
}

\author{
Masahiro Kawasaki, ${ }^{1,2}$ Kazunori Kohri, ${ }^{3,4,5}$ Takeo Moroi, ${ }^{6,2}$ and Yoshitaro Takaesu ${ }^{6,7}$ \\ ${ }^{1}$ Institute for Cosmic Ray Research, The University of Tokyo, Kashiwa 277-8582, Japan \\ ${ }^{2}$ Kavli IPMU (WPI), UTIAS, The University of Tokyo, Kashiwa 277-8583, Japan \\ ${ }^{3}$ Theory Center, IPNS, KEK, Tsukuba 305-0801, Japan \\ ${ }^{4}$ The Graduate University of Advanced Studies, Tsukuba 305-0801, Japan \\ ${ }^{5}$ Rudolf Peierls Centre for Theoretical Physics, The University of Oxford, \\ 1 Keble Road, Oxford OX1 3NP, United Kingdom \\ ${ }^{6}$ Department of Physics, The University of Tokyo, Tokyo 113-0033, Japan \\ ${ }^{7}$ Research Institute for Interdisciplinary Science, Okayama University, Okayama 700-8530, Japan
}

(Received 14 September 2017; published 8 January 2018; corrected 14 March 2019)

\begin{abstract}
We study the effects of long-lived massive particles, which decayed during the big-bang nucleosynthesis (BBN) epoch, on the primordial abundance of light elements. Compared to previous studies, (i) the reaction rates of standard $\mathrm{BBN}$ reactions are updated, (ii) the most recent observational data on the light element abundance and cosmological parameters are used, (iii) the effects of the interconversion of energetic nucleons at the time of inelastic scattering with background nuclei are considered, and (iv) the effects of the hadronic shower induced by energetic high-energy antinucleons are included. We compare the theoretical predictions on the primordial abundance of light elements with the latest observational constraints, and we derive upper bounds on the relic abundance of the decaying particle as a function of its lifetime. We also apply our analysis to an unstable gravitino, the superpartner of a graviton in supersymmetric theories, and obtain constraints on the reheating temperature after inflation.
\end{abstract}

DOI: 10.1103/PhysRevD.97.023502

\section{INTRODUCTION}

Big-bang nucleosynthesis $(\mathrm{BBN})$ is one of the most important predictions of big-bang cosmology. At a cosmic temperature of around $0.1 \mathrm{MeV}$, the typical energy of the cosmic microwave background (CMB) photon becomes sufficiently lower than the binding energies of the light elements [deuterium (D), ${ }^{3} \mathrm{He},{ }^{4} \mathrm{He}$, and so on], so the light elements can be synthesized by avoiding the dissociation due to the scattering with background photons. The cross sections for nuclear reactions governing $\mathrm{BBN}$ are well understood so that a precise theoretical calculation of the primordial light element abundance is possible with the help of numerical calculation. In addition, the primordial abundance of the light elements can be easily extracted from astrophysical observations. Comparing theoretical predictions with observational constraints, a detailed test of BBN is now possible; currently, the predicted values of

Published by the American Physical Society under the terms of the Creative Commons Attribution 4.0 International license. Further distribution of this work must maintain attribution to the author(s) and the published article's title, journal citation, and DOI. Funded by SCOAP.
$\mathrm{D}$ and ${ }^{4} \mathrm{He}$ in standard BBN (SBBN) show reasonable agreement with the observations.

It has been well recognized that, if there were to exist a new physics beyond the standard model which may induce nonstandard $\mathrm{BBN}$ reactions, the predictions of SBBN would change. ${ }^{1}$ Specifically, with long-lived unstable particles decaying into electromagnetic [13-20] or hadronic [21-32] particles, the light element abundance is affected by photodissociation, hadrodissociation, and $p \leftrightarrow n$ conversion processes. In order not to spoil the agreement between theoretical predictions and observational constraints, upper bounds on the primordial abundance of the unstable particles are obtained. Such constraints have been intensively studied in the past. Remarkably, the BBN constraints may shed light on beyond-the-standard-model particles on which collider studies cannot impose constraints. One important example is a gravitino, which is the superpartner of a graviton in supersymmetric (SUSY) models [33]. A gravitino is very weakly interacting and is produced by the scattering processes of particles in a thermal bath after inflation; the primordial abundance of a gravitino is approximately proportional to the reheating temperature after inflation. Consequently, with the

\footnotetext{
${ }^{1}$ For previous studies, see Refs. [1-12].
} 
reheating temperature being fixed, we may acquire a constraint on the gravitino mass, assuming that the gravitino is unstable. Such a constraint can be converted to the upper bound on the reheating temperature after inflation, which provides important information for studying cosmology based on SUSY models.

The purpose of this paper is to revisit the BBN constraints on long-lived particles, which we call $X$, taking into account recent progress in theoretical and observational studies of the primordial abundance of the light elements. Theoretically, understanding of the cross sections of the SBBN reactions has improved, which results in smaller uncertainties in the theoretical calculations of the light element abundance. In addition, the observational constraints on the primordial abundance of the light elements have been updated. These affect the BBN constraints on the primordial abundance of long-lived particles.

In this paper, we study the BBN constraints on the primordial abundance of long-lived exotic particles, which we parametrize by using the so-called yield variable:

$$
Y_{X} \equiv\left[\frac{n_{X}}{s}\right]_{t \ll \tau_{X}},
$$

where $n_{X}$ is the number density of $X, s$ is the entropy density, and the quantity is evaluated at the cosmic time much sooner than the lifetime of $X$ (denoted as $\tau_{X}$ ). We take into account the theoretical and observational progress. Specifically, compared to the previous studies,

(a) The reaction rates of the SBBN reactions are updated.

(b) The most recent observational constraints on the primordial abundance of the light elements are adopted.

(c) The calculation of the evolution of the hadronic showers induced by energetic nucleons from the decay is improved.

(d) We include the effect of the hadronic shower induced by energetic antinucleons from the decay. ${ }^{2}$

We consider various decay modes of long-lived particles and derive upper bounds on their abundance. We also apply our analysis to the study of the effects of an unstable gravitino on the light element abundance. In this paper, we adopt several patterns of mass spectra of superparticles (i.e., squarks, sleptons, gauginos, and Higgsinos) suggested by viable SUSY models, from which the partial decay rates of gravitino are calculated.

The organization of this paper is as follows. In Sec. II, we summarize the observational constraints on the light element abundance we adopt in our analysis. In Sec. III, we explain how the theoretical calculation of the light element abundance is performed by taking into account the effects of the decay of long-lived particles. Upper bounds

\footnotetext{
${ }^{2}$ For annihilating massive particles, we partially considered the effects induced by antinucleons emitted from the annihilation [34]
}

on the primordial abundance of generic decaying particles are given in Sec. IV. Then our analysis is applied to the case of an unstable gravitino in Sec. V. Section VI is devoted to conclusions and a discussion.

\section{OBSERVED ABUNDANCE OF THE LIGHT ELEMENTS}

We first summarize the current observational constraints on the primordial abundance of the light elements $\mathrm{D},{ }^{4} \mathrm{He}$, ${ }^{3} \mathrm{He}$, and ${ }^{7} \mathrm{Li}$. In the following, $\mathrm{A} / \mathrm{B}$ denotes the ratio of number densities of light elements $\mathrm{A}$ and $\mathrm{B}$, and the subscript $p$ indicates the primordial value.

(a) $\mathbf{D}$

The primordial abundance of D is inferred from D absorption in damped Ly- $\alpha$ systems (DLAs). Most recently, Cook et al. [35] measured D/H by observing a DLA toward QSO SDSS J1358 + 6522. Moreover, they reanalyzed four previously observed DLAs, and from the total five DLA data, they obtained the primordial $\mathrm{D}$ abundance as

$$
(\mathrm{D} / \mathrm{H})_{p}=(2.53 \pm 0.04) \times 10^{-5} .
$$

The quoted error is much smaller (by a factor of $\sim 5$ ) than those obtained in the previous study. The improvement of the $\mathrm{D}$ measurement is the main reason why we obtain more stringent BBN constraints than those in a previous work [28], as seen in later sections.

(b) ${ }^{3} \mathrm{He}$

The ${ }^{3} \mathrm{He}$ abundance is measured in protosolar objects. As described in a previous work [28], we use the ratio ${ }^{3} \mathrm{He} / \mathrm{D}$ as an observational constraint instead of ${ }^{3} \mathrm{He} / \mathrm{H}$. This is because chemical evolution can increase or decrease the ${ }^{3} \mathrm{He}$ abundance, and it is difficult to infer the primordial value for ${ }^{3} \mathrm{He}$. On the other hand, the D abundance always decreases in chemical evolution and $\mathrm{D}$ is more fragile than ${ }^{3} \mathrm{He}$. Consequently, the ratio ${ }^{3} \mathrm{He} / \mathrm{D}$ increases monotonically with time, which allows us to use the measured ${ }^{3} \mathrm{He} / \mathrm{D}$ as an upper bound on the primordial value [36]. From the ${ }^{3} \mathrm{He}$ abundance observed in protosolar clouds [37], we adopt

$$
\left({ }^{3} \mathrm{He} / \mathrm{D}\right)_{p}<0.83+0.27
$$

(c) ${ }^{4} \mathrm{He}$

The primordial mass fraction of ${ }^{4} \mathrm{He}, Y_{p}$, is inferred from the measurement of recombination lines of HeII (and HII) emitted from extragalactic HII regions. Izotov et al. [38] reported a new determination of $Y_{p}$ with the use of infrared as well as visible ${ }^{4} \mathrm{He}$ emission lines in 45 extragalactic HII regions. Their result was

$$
Y_{p}=0.2551 \pm 0.0022
$$


After Ref. [38] was published, Aver et al. [39] reanalyzed the data of Ref. [38]. They estimated the ${ }^{4} \mathrm{He}$ abundance and its error by using a Markov chain Monte Carlo (MC) analysis and obtained

$$
Y_{p}=0.2449 \pm 0.0040
$$

Thus, the two values are inconsistent, and the discrepancy is on more than a $2 \sigma$ level. If we adopt the baryon-to-photon ratio $\eta$ determined by Planck, the BBN prediction for $Y_{p}$ is consistent with Eq. (2.4), but not with Eq. (2.3). For this reason, we adopt the value given in Eq. (2.4) as a constraint on $Y_{p}$. We will also show how the constraints would change if we adopted Eq. (2.3).

(d) ${ }^{7} \mathbf{L i}\left(\right.$ and $\left.{ }^{6} \mathbf{L i}\right)$

The primordial abundance of ${ }^{7} \mathrm{Li}$ was determined by measurement of ${ }^{7} \mathrm{Li}$ in atmospheres of old metal-poor stars. The observed ${ }^{7} \mathrm{Li}$ abundance in stars with $[\mathrm{Fe} / \mathrm{H}]=-(2.5-3)$ showed an almost constant value $\left[\log _{10}\left({ }^{7} \mathrm{Li} / \mathrm{H}\right) \simeq-9.8\right]$ called the Spite plateau which was considered primordial. ${ }^{3}$ However, the plateau value turns out to be smaller than the standard BBN prediction by a factor of nearly 3. In fact, Ref. [40] reported the plateau value $\log _{10}\left({ }^{7} \mathrm{Li} / \mathrm{H}\right)=-9.801 \pm$ 0.086 , while the BBN prediction is $\log _{10}\left({ }^{7} \mathrm{Li} / \mathrm{H}\right)=$ $-9.35 \pm 0.06$ for the central value of $\eta$ suggested from the CMB data [41] (see Sec. III). This discrepancy is called the lithium problem. Moreover, the recent observation shows a much smaller ${ }^{7} \mathrm{Li}$ abundance $\left[\log _{10}\left({ }^{7} \mathrm{Li} / \mathrm{H}\right)<-10\right]$ for metal-poor stars with a metalicity below $[\mathrm{Fe} / \mathrm{H}] \sim-3[40]$. Thus, the situation regarding ${ }^{7} \mathrm{Li}$ observation is now controversial. Since we do not know of any mechanism to make ${ }^{7} \mathrm{Li}$ abundance small in such metal-poor stars, we do not use ${ }^{7} \mathrm{Li}$ as a constraint in this paper. We do not use ${ }^{6} \mathrm{Li}$, either, because ${ }^{6} \mathrm{Li}$ abundance is observed as the ratio to the number density of ${ }^{7} \mathrm{Li}$.

\section{BBN WITH DECAYING PARTICLES}

In this section, we explain how we calculate the light element abundance while taking into account the effects of decaying massive particles. Our procedure is based on that developed in Refs. [27,28], with several modifications which will be explained in the following subsections.

\section{A. Overview}

The BBN constraints strongly depend on how $X$ decays. In order to make our discussion simple, we first concentrate on the case where $X$ decays only into a particle and its antiparticle. The subsequent decays of the daughter

\footnotetext{
${ }^{3}[\mathrm{Fe} / \mathrm{H}] \equiv \log _{10}(\mathrm{Fe} / \mathrm{H})-\log _{10}(\mathrm{Fe} / \mathrm{H})_{\odot}$, where $\odot$ indicates the solar abundance.
}

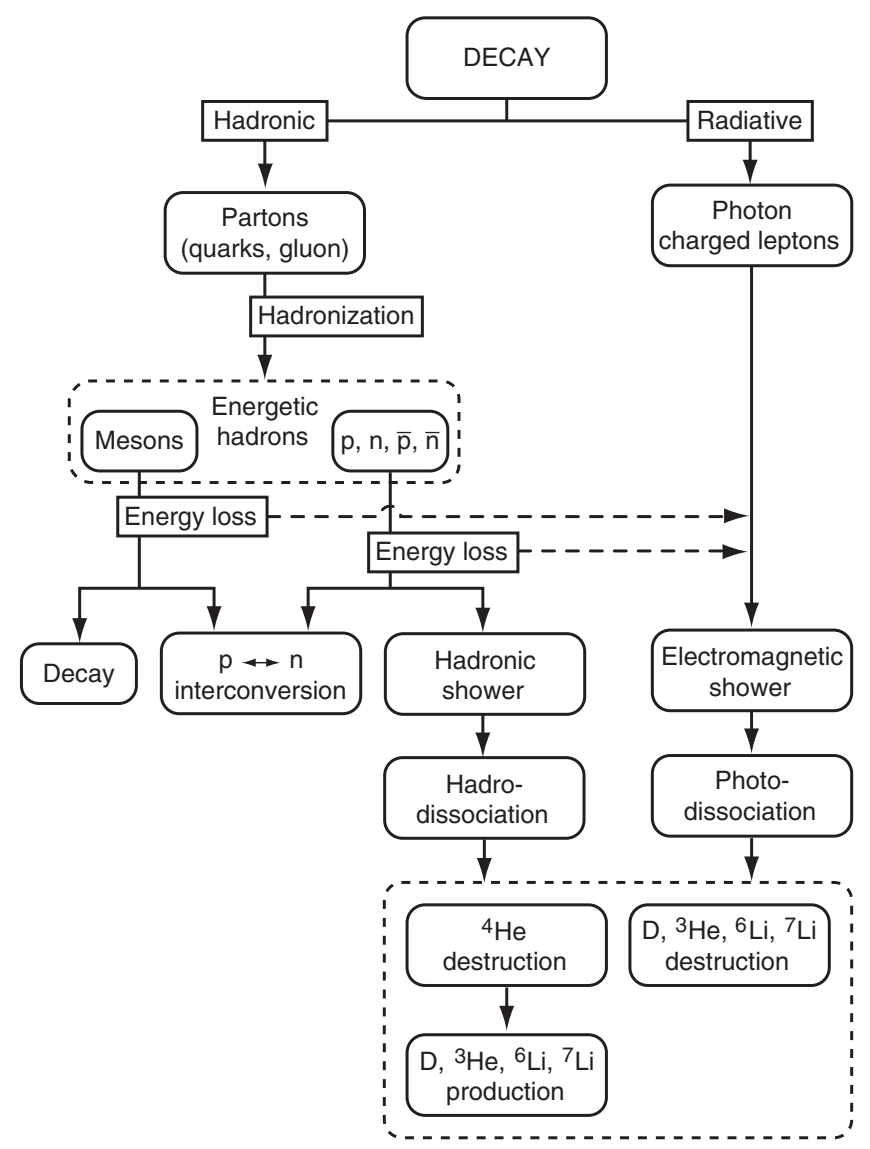

FIG. 1. Flowchart of the effects of the decay of massive particles. New effects induced by "energetic" antinucleons ( $\bar{n}$ and $\bar{p}$ ) are included in this paper.

particles from $X$ decay, as well as the hadronization processes of colored particles (if emitted), are studied using the PYTHIA 8.2 package [42]. We note here that, even if $X$ primarily decays into a pair of electromagnetically interacting particles (like $e^{+} e^{-}$or $\gamma \gamma$ pair), colored particles are also emitted by the final-state radiation; such effects are included in our paper.

There are two categories of decay processes that we encounter when studying the effects of long-lived particles on BBN:

(a) Radiative decay. With the decay of the long-lived particles, high-energy photons and electromagnetically charged particles are emitted.

(b) Hadronic decay. High-energy colored particles (i.e., quarks or gluons) are emitted in the decay.

We give a first overview of them in the following. (A flowchart of the effects induced by the decay of $X$ is given in Fig. 1.)

\section{Radiative decay modes}

Energy injections by energetic electromagnetic particles induce electromagnetic showers through their scattering off the background photons and electrons [11,13-18,26,30,32]. 


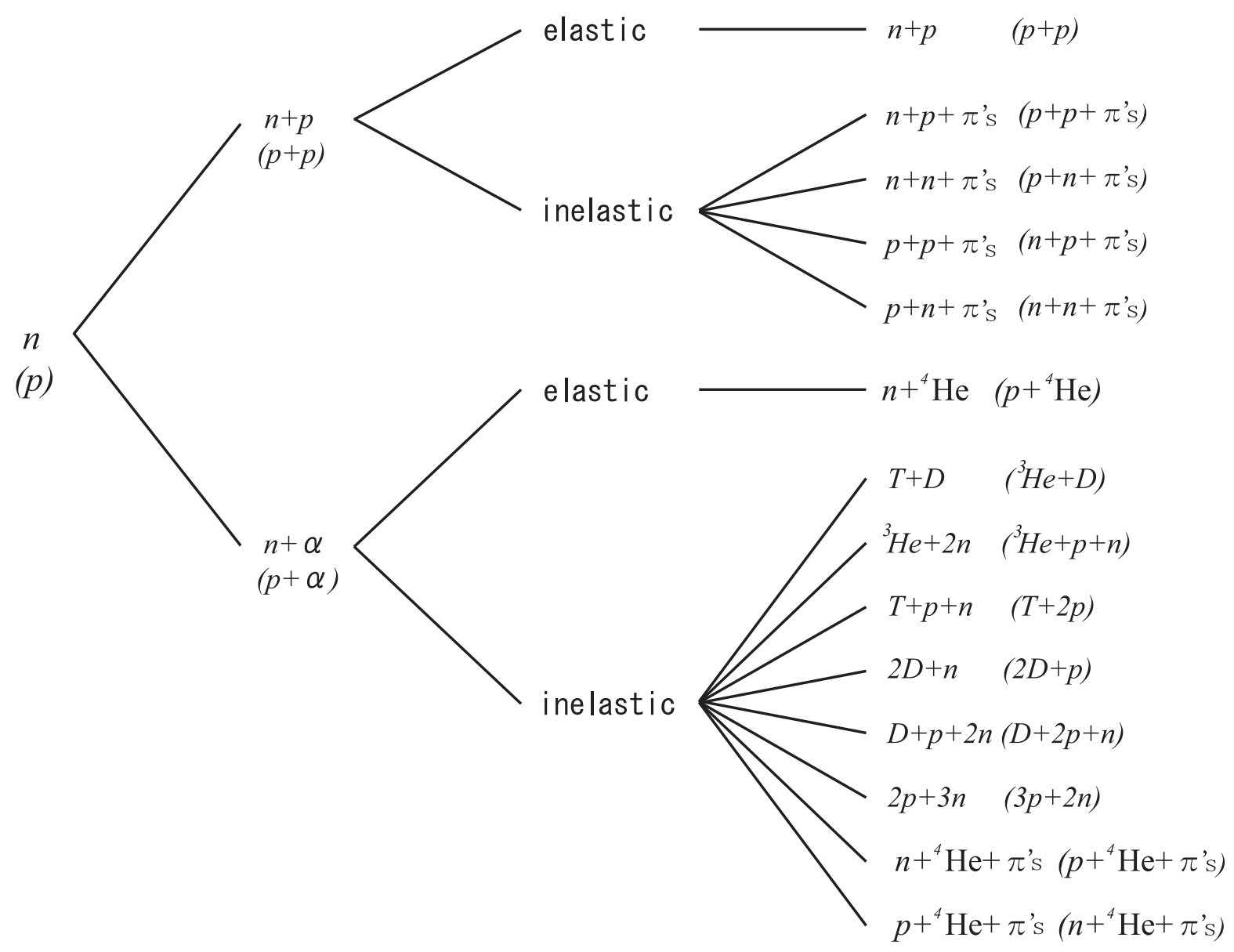

FIG. 2. Schematic picture of a hadronic shower induced by a high-energy projectile $n$ (or $p$ ) which scatters off the background proton or the background ${ }^{4} \mathrm{He}$. (Here, $T$ denotes tritium.)

The electromagnetic particles include $\gamma$ and $e^{ \pm}$, as well as charged hadrons. Energetic photons in the shower can destroy the light elements, particularly $\mathrm{D}$ and ${ }^{4} \mathrm{He}$, produced by the SBBN reactions. (Such processes are called photodissociation.) The photon spectrum in the electromagnetic shower is determined by the total amount of visible energy injected by the decay and the temperature of the background thermal bath $[11,13,14]$; for example, at high precision, the normalization of the photon spectrum is proportional to the total amount of energy injection. In our numerical calculation, the total visible energy, which is the sum of the energies of the photon and the charged particles after hadronization, is calculated, from which the normalization of the photon spectrum is determined.

The photodissociation processes become effective when the threshold energy for the $e^{+} e^{-}$pair creation by the scattering of high-energy photons and background photons, which is approximately given by $E_{\mathrm{th}}^{(\gamma)} \sim m_{e}^{2} / 22 T$ (with $m_{e}$ and $T$ being the electron mass and the cosmic temperature, respectively) [13], is larger than the threshold energy for the dissociation processes of the light elements. For the photodissociation of ${ }^{4} \mathrm{He}$, this is the case at cosmic temperature lower than $\sim 1 \mathrm{keV}$ (which corresponds to the cosmic time $\left.t \gtrsim 10^{6} \mathrm{sec}\right)$. With the photodissociation of ${ }^{4} \mathrm{He}$, both ${ }^{3} \mathrm{He}$ and $\mathrm{D}$ are copiously produced. Thus, for a long-lived particle with a lifetime longer than $\sim 10^{6} \mathrm{sec}$, the D and ${ }^{3} \mathrm{He}$ abundance gives stringent constraints on the radiatively decaying modes. The photodissociation of $\mathrm{D}$ becomes effective at higher temperature because of the smallness of its binding energy; the photodissociation of $\mathrm{D}$ may be important for a long-lived particle with a lifetime longer than $\sim 10^{4} \mathrm{sec}$, at which point significant destruction of D takes place $[15,17]$. Another effect of the electromagnetic shower on BBN is the nonthermal production of ${ }^{6} \mathrm{Li}$. Energetic forms of $\mathrm{T}$ and ${ }^{3} \mathrm{He}$ are produced through photodissociation of ${ }^{4} \mathrm{He}$, and they scatter off the background ${ }^{4} \mathrm{He}$ and synthesize ${ }^{6} \mathrm{Li}$. The production of ${ }^{7} \mathrm{Li}$ and ${ }^{7} \mathrm{Be}$ due to energetic ${ }^{4} \mathrm{He}$ scattered by energetic photons through inelastic $\gamma+{ }^{4} \mathrm{He}$ is negligible.

\section{Hadronic decay modes}

In hadronic decays, the emitted colored particles fragment into hadrons such as pions, kaons, nucleons (i.e., neutron $n$ and proton $p$ ), and antinucleons (i.e., antineutron $\bar{n}$ and antiproton $\bar{p})$. Hereafter, $N=p$ or $n(\bar{N}=\bar{p}$ or $\bar{n})$ 


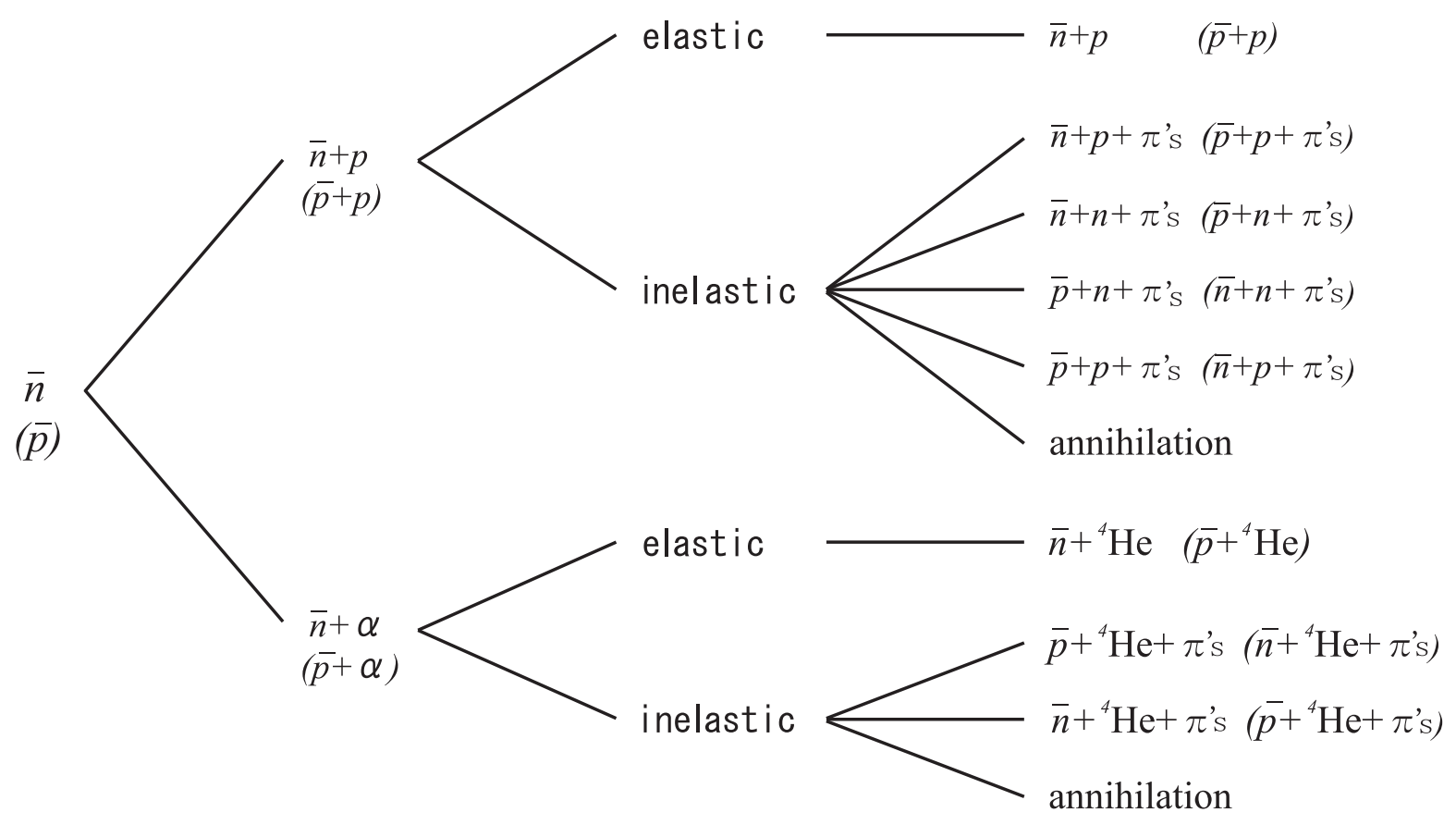

FIG. 3. The same as Fig. 2, but for a high-energy projectile $\bar{n}$ (or $\bar{p}$ ).

denotes the nucleon (antinucleon). Energetic hadrons, particularly nucleons, induce hadronic showers and hadrodissociation processes. (The effect of the Lorentz suppression of the decay rate is included for energetic unstable hadrons.) In addition, even after being stopped, some of the hadrons (particularly charged pions and nucleons) change the neutron-to-proton ratio in the background plasma, which affects the ${ }^{4} \mathrm{He}$ and $\mathrm{D}$ abundance. The most important effects of the hadronic decay modes are summarized as follows:

(a) When a massive particle decays into hadrons at $T \gtrsim$ $\mathcal{O}(0.1) \mathrm{MeV}$ [i.e., when the lifetime is shorter than $\sim \mathcal{O}\left(10^{2}\right)$ sec], the high-energy hadrons are stopped in the thermal plasma [28]. The extra pions and nucleons affect the neutron-to-proton ratio after the neutron freeze-out by interchanging backgrounds $p$ and $n$ through strongly interacting interconversion processes like

$$
\begin{aligned}
& \pi^{+}+n \rightarrow p+\pi^{0}, \\
& \pi^{-}+p \rightarrow n+\pi^{0} .
\end{aligned}
$$

There also exist similar interconversion processes caused by injected nucleons. The neutron-to-proton ratio $n / p$ increases due to the strongly interacting conversions, resulting in the increase of the abundance of ${ }^{4} \mathrm{He}$ and $\mathrm{D}[21,25]$.

(b) If the background temperature at the time of the decay is low enough, the emitted hadrons cannot be stopped in the plasma; this is the case for energetic $n$ and $\bar{n}$ ( $p$ and $\bar{p})$ if $T \lesssim 0.1 \mathrm{MeV}(T \lesssim 30 \mathrm{keV})$ [28]. The energetic nucleons scatter off and destroy the background nuclei [22-24]. The processes considered in this paper are summarized in Figs. 2 and 3. Specifically, through the destruction of ${ }^{4} \mathrm{He}$ by high-energy neutrons, an overproduction of D may occur, which leads to a stringent constraint on the primordial abundance of the hadronically decaying long-lived particles [27,28,31,34]. In addition, energetic ${ }^{3} \mathrm{He}$, ${ }^{4} \mathrm{He}$, and $\mathrm{T}$ produce ${ }^{7} \mathrm{Li},{ }^{7} \mathrm{Be}$, and ${ }^{6} \mathrm{Li}$ nonthermally through scattering off the background ${ }^{4} \mathrm{He}$.

\section{B. New implementations in the numerical calculation}

As mentioned before, our analysis is based on Refs. [27,28]. Here, we summarize the new implementations in the numerical calculation added after Refs. [27,28,31].

(a) We update the SBBN reaction rates and their uncertainties.

(b) We revise the algorithm to calculate the evolution of the hadronic shower induced by the injections of energetic $p$ and $n$. Specifically, we include the $p \leftrightarrow n$ interconversion of the energetic nucleons via inelastic scattering. ${ }^{4}$ In the inelastic scattering

\footnotetext{
${ }^{4}$ The interconversion of energetic $p$ and $n$ should not be confused with the interconversion of background $p$ and $n$. The former affects the stopping rate of the energetic nucleons, which induces hadronic showers as well as hadrodissociation processes. On the contrary, the latter affects the neutron-to-proton ratio after the neutron freeze-out to which $Y_{p}$ is sensitive; the effect of the latter was already taken into account in the previous analysis $[25,27,28,31]$.
} 
processes of energetic nucleon (i.e., $p, n, \bar{p}$, or $\bar{n}$ ) with the background nuclei, it is likely that the projectile nucleon carries away most of the energy, while other final-state particles are less energetic; the kinetic energies of the final-state particles other than the most energetic one are typically $K_{T} \sim 140 \mathrm{MeV}$. The conversion of the projectile nucleon via the inelastic scattering affects the (effective) energy-loss rate of the nucleon, as we discuss below.

(c) We newly include the effects of energetic $\bar{p}$ and $\bar{n}$ injections by the decay.

\section{1. $S B B N$ reactions}

Making a comparison to previous studies [28,31], we update the SBBN reaction rates by adopting those given in Refs. $[43,44]$, which are obtained by fitting relatively new experimental data. In order to account for uncertainties in the reaction rates, we perform MC simulations to estimate error propagations for the light element abundance. For given values of the lifetime and the primordial abundance of $X$, we perform 1000 runs of the calculation of the light element abundance with the assumption that the reaction rates (as well as other parameters in the calculation) are random Gaussian variables. In each run, the reaction rate $R_{i}$ for the $i$ th reaction is determined as

$$
R_{i}(T)=\bar{R}_{i}(T)+\sigma_{i}(T) y_{i},
$$

where $\bar{R}_{i}$ and $\sigma_{i}$ are the central value and the standard deviation of the reaction $i$, and $y_{i}$ is the temperatureindependent Gaussian variable with the probability distribution

$$
P(y)=\frac{1}{\sqrt{2 \pi}} \exp \left(-\frac{1}{2} y^{2}\right) .
$$

Notice that, as is explicitly shown in Eq. (3.3), some of the uncertainties of the reaction rates depend on the cosmic temperature. Throughout each run, such a reaction rate is determined by adding the temperature-dependent uncertainty multiplied by the temperature-independent random Gaussian variable to the central value. If the upper and lower uncertainties of a reaction rate are asymmetric, we take upward and downward fluctuations with equal probability, assuming that they obey one-sided Gaussian distributions.

In order to check on the consistency between the observational constraints summarized in Sec. II and the SBBN predictions, we compare them in Fig. 4; we plot the SBBN values of the light element abundance as functions of the baryon-to-photon ratio $\eta=n_{\mathrm{B}} / n_{\gamma}$. From the top to the bottom, we plot (i) the mass fraction of ${ }^{4} \mathrm{He}$ $\left(Y_{p}\right)$, (ii) the deuterium-to-hydrogen ratio $(\mathrm{D} / \mathrm{H})$, (iii) the ${ }^{3} \mathrm{He}$-to-hydrogen ratio $\left({ }^{3} \mathrm{He} / \mathrm{H}\right)$, (iv) the ${ }^{7} \mathrm{Li}$-to-hydrogen ratio $\left({ }^{7} \mathrm{Li} / \mathrm{H}\right)$, and $(\mathrm{v})$ the ${ }^{3} \mathrm{He}$-to-deuterium ratio $\left({ }^{3} \mathrm{He} / \mathrm{D}\right)$.

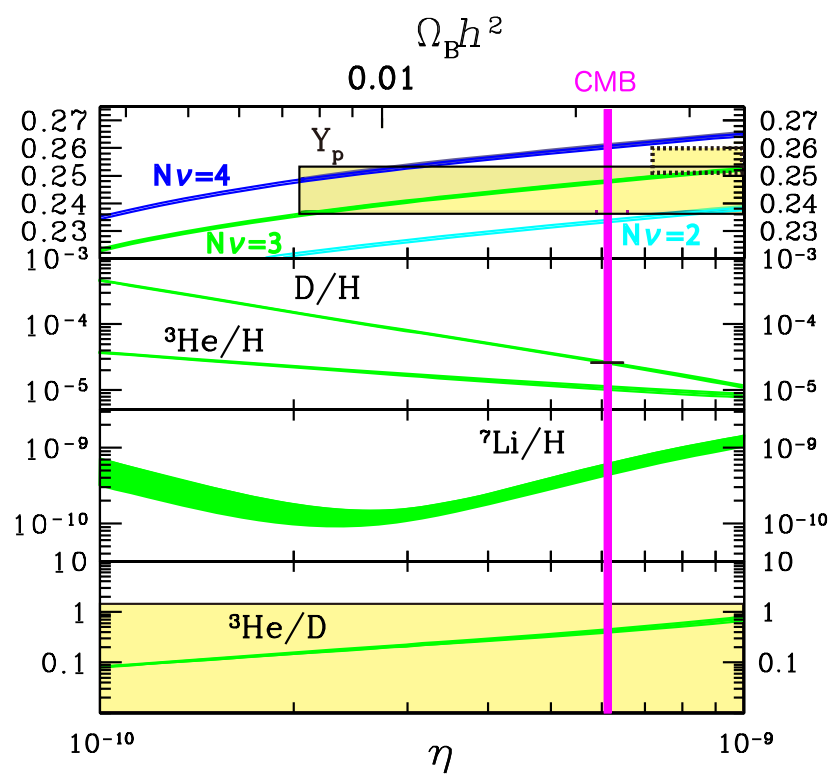

FIG. 4. Theoretical predictions of the light element abundance as functions of the baryon-to-photon ratio $\eta=n_{\mathrm{B}} / n_{\gamma}$. (The upper horizontal axis shows $\Omega_{\mathrm{B}} h^{2}$, where $\Omega_{\mathrm{B}}$ is the density parameter of baryon and $h$ is the Hubble constant in units of $100 \mathrm{~km} / \mathrm{sec} / \mathrm{Mpc}$.) The vertical band shows $\eta=(6.11 \pm$ $0.08) \times 10^{-10}$, which is the $2 \sigma$ band of the baryon-to-photon ratio suggested by the CMB observations [41]. For $Y_{p}$, we plot results for an effective number of neutrino species $N_{\nu}=2,3$, and 4 (from bottom to top). The boxes indicate the observational values with $2 \sigma$ uncertainties. [For $Y_{p}$, the boxes surrounded by the solid and dashed lines correspond to Eq. (2.4) and (2.3), respectively.]

The theoretical predictions show $2 \sigma$ bands due to uncertainties in the experimental data of the cross sections and lifetimes of the nuclei. The boxes indicate the $2 \sigma$ observational constraints (see Sec. II). The CMB observations provide independent information about the baryon-tophoton ratio; we adopt

$$
\eta=(6.11 \pm 0.04) \times 10^{-10} \quad(68 \% \text { C.L. }),
$$

which is based on the TT, TE, EE + lowP + BAO analysis of the Planck Collaboration, $\Omega_{\mathrm{B}} h^{2}=0.02229_{-0.00027}^{+0.00029}$ (95\% C.L.), where $\Omega_{\mathrm{B}}$ is the density parameter of baryon and $h$ is the Hubble constant in units of $100 \mathrm{~km} / \mathrm{sec} / \mathrm{Mpc}$ [41]. In Fig. 4, we also show the CMB constraint on $\eta$ at $2 \sigma$ (the vertical band). We can see that the SBBN predictions based on the baryon-to-photon ratio suggested from the $\mathrm{CMB}$ observations are in reasonable agreement with the observations.

\section{High-energy $n$ and $p$ injections}

The effects of the high-energy $n$ and $p$ injected into the thermal bath were studied in Refs. [22-24,27,28]. Making a comparison to the previous studies, we newly include the 
interconversion reactions between energetic $p$ and $n$ at the time of the inelastic scattering that is accompanied by pion emission (see Fig. 2). Once an energetic $p$ or $n$ is injected into thermal plasma, it can be converted to $n$ or $p$ through inelastic scattering off the background nuclei, i.e., $p$ or ${ }^{4} \mathrm{He}$. (Hereafter, the background ${ }^{4} \mathrm{He}$ is denoted as $\alpha_{\mathrm{BG}}$.) As we discuss below, such an interconversion affects the (effective) energy-loss rates of nucleons during their propagation in thermal plasma, resulting in a change of the hadrodissociation rates.

The effects of the interconversion of energetic $p$ and $n$ are important when the cosmic temperature becomes lower than $\sim 0.1 \mathrm{MeV}$. This is because the energetic nucleons are stopped in the thermal plasma if the background temperature is high enough; as we have mentioned, the neutron is likely to be stopped when $T \gtrsim 0.1 \mathrm{MeV}$, while the proton is stopped when $T \gtrsim 30 \mathrm{keV}$. Once stopped, the nucleons do not induce hadrodissociation processes. An energetic neutron, which would have a sufficient time to induce hadronic showers without interconversion, does not cause hadronic showers when it is converted to a proton. Thus, the inclusion of the energetic $p \leftrightarrow n$ conversion makes the BBN constraints milder, particularly when $30 \mathrm{keV} \lesssim T \lesssim$ $0.1 \mathrm{MeV}$ (i.e., $10^{2} \mathrm{sec} \lesssim t \lesssim 10^{3} \mathrm{sec}$ ). Inelastic scattering occurs with the targets of $p$ and $\alpha_{\mathrm{BG}}$. The interconversion rates are estimated as follows:

(a) Because of the lack of experimental data on a cross section of each mode for the $n$ (projectile) $+p$ (target) and $p$ (projectile $)+p$ (target) processes shown in Fig. 2, we assume that all of the cross sections for those eight modes $\left(n+p, p+p \rightarrow p+p+\pi^{\prime} \mathrm{s}\right.$, $p+n+\pi^{\prime} \mathrm{s}, n+p+\pi^{\prime} \mathrm{s}, n+n+\pi^{\prime} \mathrm{s}$ ) are equal. (In the case of $p+p \rightarrow n+n+\pi^{\prime} \mathrm{s}$, at least two pions should be emitted, and hence we consider the process only if it is kinematically allowed.) In addition, we neglect the effects of interconversion induced by the emitted pions from the inelastic scattering. That is because those pions decay before they scatter off the background particles.

(b) With inelastic $n+\alpha_{\mathrm{BG}}$ scattering, the energetic $n$ can be again converted to $p$. Because of the lack of experimental data, we assume that, for inelastic processes with pion emission, the cross section for $n+$ ${ }^{4} \mathrm{He}$ scattering is equal to that for $p+{ }^{4} \mathrm{He}$ scattering. Since the rates of the inelastic scattering $n+{ }^{4} \mathrm{He} \rightarrow$ $n+{ }^{4} \mathrm{He}$ and $p+{ }^{4} \mathrm{He}$ are relatively small [45], effects of the interconversion due to the inelastic $n+\alpha_{\mathrm{BG}}$ scattering are unimportant and do not change the hadronic shower evolution.

We also comment on the nonthermal production processes of ${ }^{6} \mathrm{Li},{ }^{7} \mathrm{Li}$, and ${ }^{7} \mathrm{Be}$. With the inelastic scattering $N+\alpha_{\mathrm{BG}} \rightarrow \cdots$, the final state may contain energetic $\mathrm{T}$, ${ }^{3} \mathrm{He}$, and ${ }^{4} \mathrm{He}$. They may scatter off $\alpha_{\mathrm{BG}}$ to produce heavier elements, i.e., ${ }^{6} \mathrm{Li},{ }^{7} \mathrm{Li}$, and ${ }^{7} \mathrm{Be}[22-24,28]$. Although we do not use ${ }^{6} \mathrm{Li}$ and ${ }^{7} \mathrm{Li}$ to constrain the primordial abundance of
$X$, these nonthermal production processes are included in our numerical calculation. For example, we include the processes ${ }^{4} \mathrm{He}+\alpha_{\mathrm{BG}} \rightarrow{ }^{6} \mathrm{Li}+\cdots,{ }^{7} \mathrm{Li}+\cdots$, and ${ }^{7} \mathrm{Be}+\cdots$, whose effects were not taken into account in Ref. [28]. For the study of these processes, the energy distribution of ${ }^{4} \mathrm{He}$ produced by inelastic hadronic scattering is necessary. We determine energy distribution of the final-state ${ }^{4} \mathrm{He}$ using the prescription given in Appendix C of Ref. [28]. Notice that, for the nonthermal production processes of ${ }^{6} \mathrm{Li}$ induced by $\mathrm{T}$ and ${ }^{3} \mathrm{He}$, the energy distributions of $\mathrm{T}$ and ${ }^{3} \mathrm{He}$ produced by the hadrodissociation of $\alpha_{\mathrm{BG}}$ are obtained by fitting the experimental data (see Ref. [28]).

\section{Injections of energetic $\bar{n}$ or $\bar{p}$}

In this paper, we newly include the effects of the injections of the energetic antinucleons $\bar{n}$ or $\bar{p}$. We consider only the scattering of energetic antinucleons off the background $p$ and $\alpha_{\mathrm{BG}}$, and the antinucleons are treated as a source of hadronic showers. ${ }^{5}$ The high-energy nucleons in the hadronic showers induced by $\bar{n}$ and $\bar{p}$ can destroy $\alpha_{\mathrm{BG}}$ and, further, produce copious high-energy daughter nuclei. In Fig. 3, we show a schematic picture for the reactions induced by energetic antinucleons. Because of the lack of experimental data for the processes including the antinucleons, we adopt several approximations and assumptions on the reactions induced by the energetic antinucleon. We note here that, in adopting approximations or assumptions, we require that the constraints become conservative in order not to overconstrain the properties of long-lived particles.

First, let us consider the scattering of energetic antinucleons with the background $p$. We use the experimental data of the differential cross sections for the $\bar{p}$ - $p$ scattering by referring to Ref. [46] for the total and elastic cross sections and to Ref. [47] for the annihilation cross sections. Because of the lack of experimental data, we use the data for the $\bar{p}-p$ scattering to estimate the cross sections for other processes. (i) We assume that the differential cross sections for $\bar{n}-p$ scattering are the same as those for the corresponding $\bar{p}-p$ scattering paired in Fig. 3. We expect this assumption to be reasonable because the Coulomb corrections to the cross sections are estimated to be less than a few percent for the energy of our interest [28]. (ii) Through inelastic scattering off the background $p$, the strongly interacting $p \leftrightarrow n$ interconversion reactions occur with emitting pions. Then there are four possible combinations of final-state antinucleon and scattered nucleon; the final-state antinucleon may be $\bar{p}$ or $\bar{n}$, while the nucleon may be $p$ or $n$ (see Fig. 3). We assume the differential cross sections for these processes to be equal and use the data for the $\bar{p}-p$ scattering for all of these processes because the

\footnotetext{
${ }^{5}$ On the other hand, for antinucleons stopped in the plasma, we explained their effects on the interconversion reactions between background $n$ and $p$ earlier in the paper.
} 
cross sections for some of the final states are unknown. Here, one remark is that the process $\bar{n}$ (projectile) + $p$ (target) $\rightarrow \bar{p}+n$ needs at least two pion emission, so we take it into account when the process is kinematically allowed.

Next, we discuss the scattering of energetic antinucleons off $\alpha_{\mathrm{BG}}$. Unfortunately, experimental data on the cross sections for the scattering processes of an antinucleon with ${ }^{4} \mathrm{He}$ are insufficient to perform a detailed study. Therefore, we consider only the energy loss and interconversion of an antinucleon induced by inelastic scattering with $\alpha_{\mathrm{BG}}$. Approximating that the energetic antinucleon scatters off individual nucleons in ${ }^{4} \mathrm{He}$, the cross sections for the scattering processes with ${ }^{4} \mathrm{He}$ are taken to be 4 times larger than those with the background nucleons. Notice that this assumption is reasonable when the energy of $\bar{N}$ is larger than the binding energy of ${ }^{4} \mathrm{He} .{ }^{6}$ In addition, for the scattering induced by the antinucleon, we neglect the subsequent reactions induced by the cascade products from $\alpha_{\mathrm{BG}}$; in other words, we neglect the destruction of $\alpha_{\mathrm{BG}}$ and the recoil energy of $\alpha_{\mathrm{BG}}$ (if it is not destroyed). These would give the most conservative bounds.

For annihilation reactions of an antinucleon with a background $p$ or $\alpha_{\mathrm{BG}}$, we expect emission of energetic hadrons, by which the light element abundance should be affected. However, because we do not have sufficient data on differential cross sections, we conservatively neglect any effects after the annihilation of antinucleon. Thus, in our calculation, the effect of the pair annihilations of the antinucleon is only to reduce the number of energetic antinucleons.

\section{4. $\xi$ parameters}

In this paper, the effects of hadrodissociation are parametrized by functions called $\xi_{A_{i}}$ (with $A_{i}$ being $n, \mathrm{D}, \mathrm{T},{ }^{3} \mathrm{He}$, $\alpha,{ }^{6} \mathrm{Li},{ }^{7} \mathrm{Li}$, and $\left.{ }^{7} \mathrm{Be}\right)[22-24,27,28]$. For $A_{i} \neq \alpha, \xi_{A_{i}}$ is the total number of $A_{i}$ values produced by the hadronic decay of one parent particle $X$, while $\xi_{\alpha}$ is the total number of $\alpha_{\mathrm{BG}}$ values destroyed. With the properties of the long-lived particle $X$ given, $\xi_{A_{i}}$ values depend on three quantities: the cosmic temperature $T$, the mass fraction of ${ }^{4} \mathrm{He}$, and the baryon-to-photon ratio $\eta$.

It is notable that $\xi_{A_{i}}$ is defined as the value just after the shower evolutions. Notice that the time scale of the shower evolution is much shorter than those of any nuclear reactions in SBBN. Therefore, the face value of $\xi_{A_{i}}$ does not represent a net increase or decrease of the nuclei $A_{i}$. For example, ${ }^{6} \mathrm{Li}$ produced by the nonthermal

\footnotetext{
${ }^{6}$ For example, see the plots shown in Ref. [46], in which the cross section of the process $\bar{p}+\mathrm{D}$ is approximately 2 times larger than that of $\bar{p}+n$ for a high-energy $\bar{p}$. Similarly, it is also known that the cross sections of $\bar{p}+{ }^{4} \mathrm{He}$ are approximately 4 times larger than that of $\bar{p}+n$ at high energies.
}

reactions may be further processed in the $\mathrm{SBBN}$ reaction as ${ }^{6} \mathrm{Li}+p \rightarrow{ }^{3} \mathrm{He}+{ }^{4} \mathrm{He}$.

In our case of interest, the hadronic showers are triggered by the injections of high-energy nucleons (i.e., $N=p$ and $n$ ) and antinucleons (i.e., $\bar{N}=\bar{p}$ and $\bar{n}$ ) which originate from the hadronizations of colored particles emitted during the decay of long-lived particles. $\xi_{A_{i}}$ value are given by the convolutions of two functions as

$\xi_{A_{i}}\left(T, Y_{p}, \eta\right)=\sum_{N=n, \bar{n}, p, \bar{p}} \int \tilde{\xi}_{A_{i}, N}\left(E_{N}, T, Y_{p}, \eta\right) \frac{d n_{N}}{d E_{N}} d E_{N}$,

where $\left(d n_{N} / d E_{N}\right)$ is the energy spectrum of the injected nucleon $N$ with energy $E_{N}$ per a single massive particle decay, while $\tilde{\xi}_{A_{i}, N}$ is the number of the light element $A_{i}$ produced (or destroyed, for $A_{i}=\alpha$ ) by the products of a hadronic shower induced by the injection of a single $N$ with energy $E_{N}$. We calculate the functions $\tilde{\xi}_{A_{i}, N}$ based on the procedure explained in Ref. [28]. ${ }^{7}$

In Figs. 5-9, we show $\tilde{\xi}_{n, N}, \tilde{\xi}_{\mathrm{D}, N}, \tilde{\xi}_{\mathrm{T}, N}, \tilde{\xi}_{3 \mathrm{He}, N}$, and $\tilde{\xi}_{\alpha, N}$, respectively, as functions of the kinetic energy $E_{N}$ for $N=n, \bar{n}, p$, and $\bar{p}$, taking $T=4 \mathrm{keV}$. Notice that, at $T=4 \mathrm{keV}$, the energetic neutrons with $E_{n}>\mathcal{O}(1) \mathrm{TeV}$ lose their kinetic energy immediately down to $\sim 1 \mathrm{TeV}$ [31]. Thus, the $\tilde{\xi}_{\alpha, N}$ values become insensitive to $E_{N}$ for $E_{N} \gtrsim 1 \mathrm{TeV}$. By the same token, the emitted high-energy protons with $E_{n}>\mathcal{O}(10) \mathrm{GeV}$ lose their kinetic energy down to $\sim 10 \mathrm{GeV}$. The figures also show how the $\tilde{\xi}_{A_{i}, N}$ parameters depend on the cosmic temperature, taking $E_{N}=1 \mathrm{TeV}$. As one can see, $\tilde{\xi}_{A_{i}, N}$ parameters increase as the temperature decreases until $T \sim 0.5 \mathrm{keV}$; this is because the mean free paths of energetic nucleons lengthen as the temperature drops. We can also see sharp dropoffs of the $\tilde{\xi}$ parameters at $T \sim 0.5 \mathrm{keV}$; they come from the fact that, when the cosmic temperature is lower than $\sim 0.5 \mathrm{keV}$, the energetic neutrons decay before scattering off the background nuclei and the neutron contributions to the hadrodissociation become negligible.

From the figures, we find that the injections of $\bar{n}$ and $\bar{p}$ should change the total number of the destroyed $\alpha_{\mathrm{BG}}$ by $\sim 20 \%-30 \%$. As we will show later, the constraints become stronger by $10 \%-30 \%$ when we include the

\footnotetext{
${ }^{7}$ In the calculation of $\xi_{6 \mathrm{Li}}$ in Ref. [28], there was an error in numerical calculation of the Coulomb stopping powers of energetic $\mathrm{T}$ and ${ }^{3} \mathrm{He}$, with which $\xi_{6 \mathrm{Li}}$ was maximized at the cosmic temperature of $\sim 40-50 \mathrm{keV}$. The error was corrected in subsequent studies, e.g., in Ref. [48], and $\xi_{6 \mathrm{Li}}$ is peaked at the cosmic temperature of $T \sim 20 \mathrm{keV}$. However, the error did not affect the resultant value of relic ${ }^{6} \mathrm{Li}$ abundance because ${ }^{6} \mathrm{Li}$, if it exists, is completely destructed by the process of ${ }^{6} \mathrm{Li}+p \rightarrow{ }^{3} \mathrm{He}+{ }^{4} \mathrm{He}$. We thank K. Jedamzik for his comments on this issue.
} 

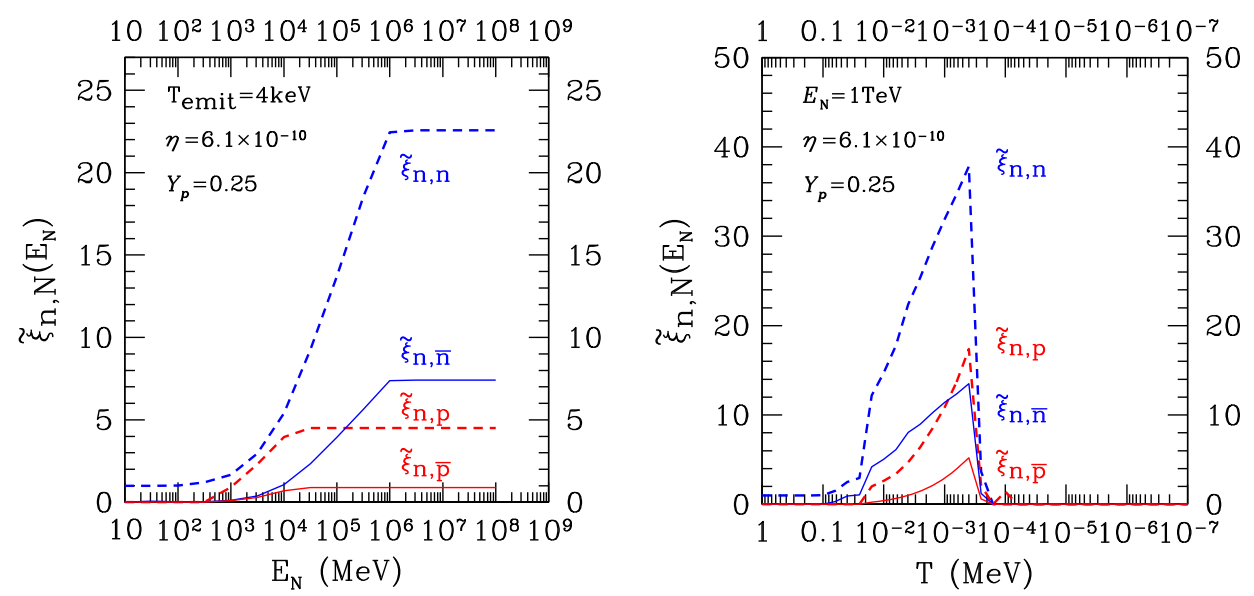

FIG. 5. $\quad \tilde{\xi}_{n, N}$ values for $T=4 \mathrm{keV}$ as functions of the kinetic energy (left panel) and those for $E_{N}=1 \mathrm{TeV}$ as functions of the cosmic temperature (right panel). Here, $N=n, \bar{n}, p$, and $\bar{p}$, and we have $\eta=6.1 \times 10^{-10}$ and $Y_{p}=0.25$.
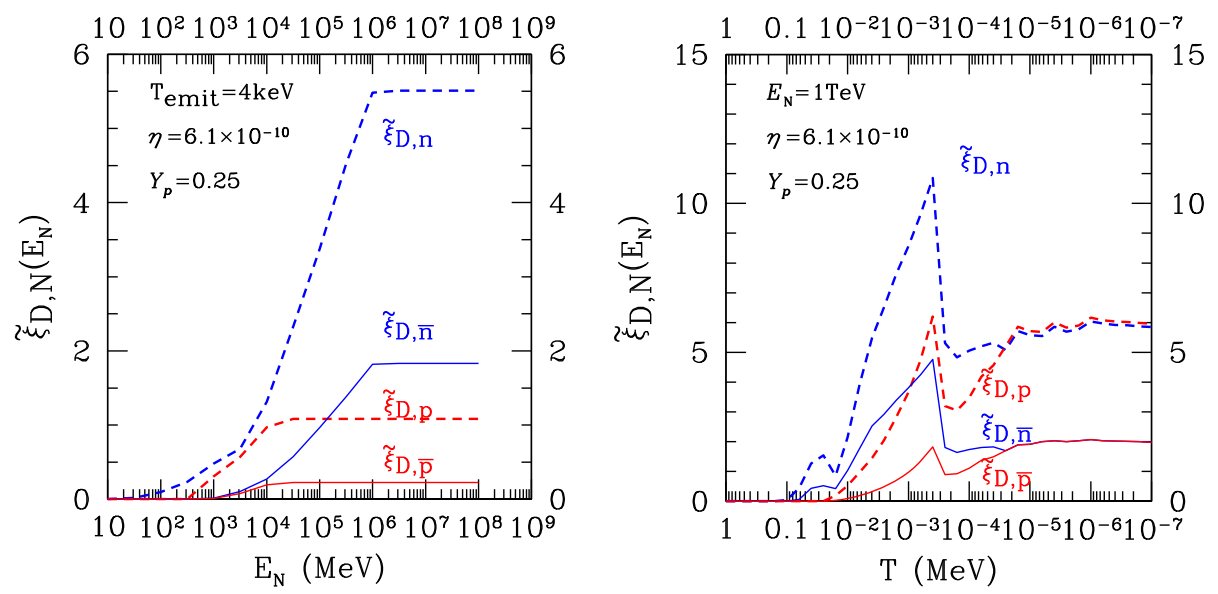

FIG. 6. $\tilde{\xi}_{\mathrm{D}, N}$ values for $T=4 \mathrm{keV}$ as functions of the kinetic energy (left panel) and those for $E_{N}=1 \mathrm{TeV}$ as functions of the cosmic temperature (right panel). Here, $N=n, \bar{n}, p$, and $\bar{p}$, and we have $\eta=6.1 \times 10^{-10}$ and $Y_{p}=0.25$.
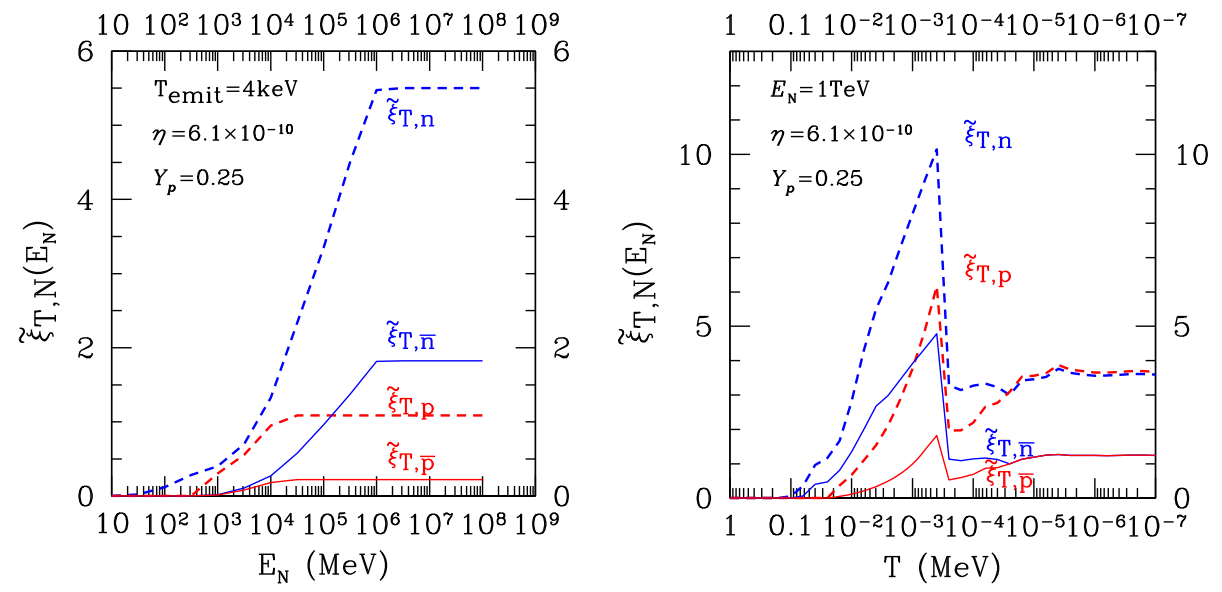

FIG. 7. $\tilde{\xi}_{\mathrm{T}, N}$ values for $T=4 \mathrm{keV}$ as functions of the kinetic energy (left panel) and those for $E_{N}=1 \mathrm{TeV}$ as functions of the cosmic temperature (right panel). (In the subscript of $\tilde{\xi}_{\mathrm{T}, N}$, T denotes tritium.) Here, $N=n, \bar{n}, p$, and $\bar{p}$, and we have $\eta=6.1 \times 10^{-10}$ and $Y_{p}=0.25$. 

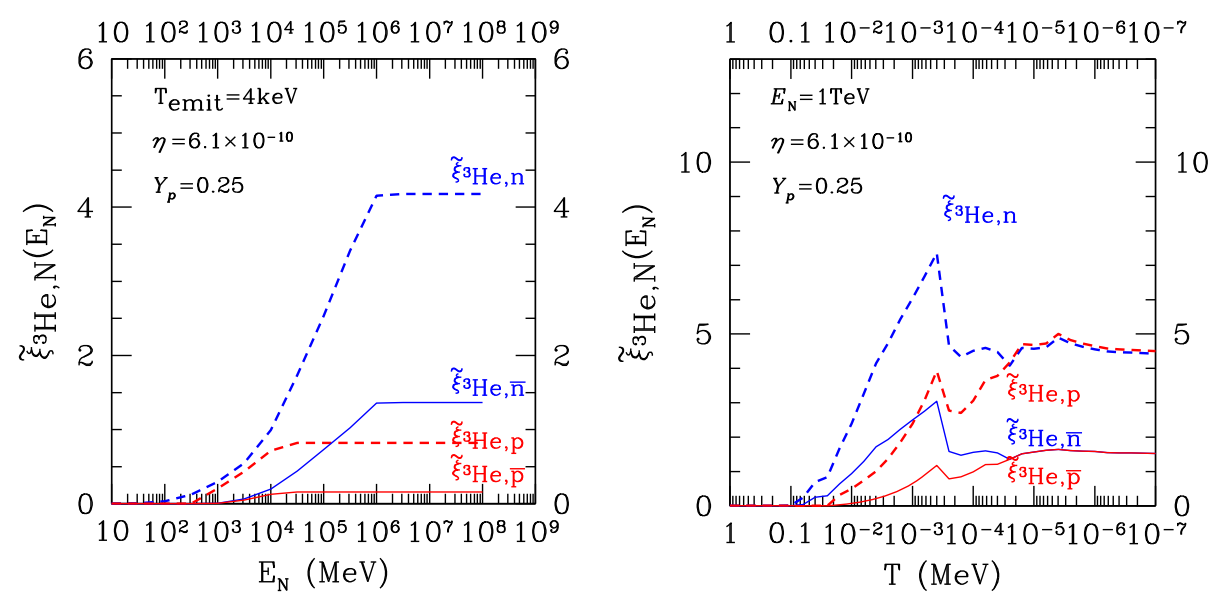

FIG. 8. $\tilde{\xi}_{3 \mathrm{He}, N}$ values for $T=4 \mathrm{keV}$ as functions of the kinetic energy (left panel) and those for $E_{N}=1 \mathrm{TeV}$ as functions of the cosmic temperature (right panel). Here, $N=n, \bar{n}, p$, and $\bar{p}$, and we have $\eta=6.1 \times 10^{-10}$ and $Y_{p}=0.25$.
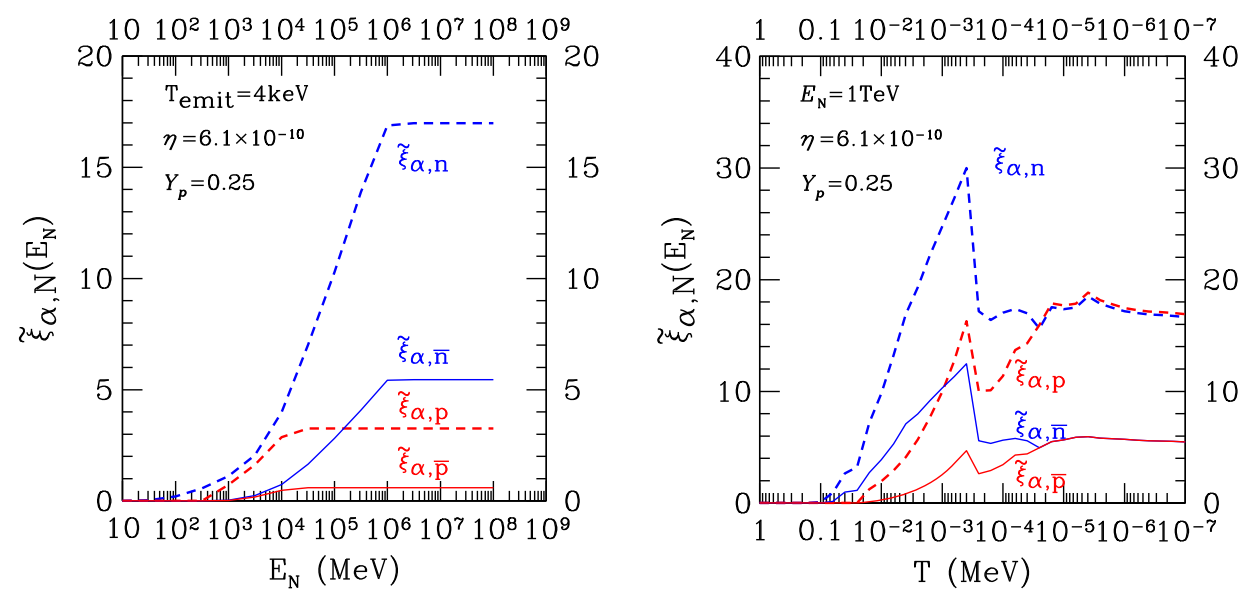

FIG. 9. $\tilde{\xi}_{\alpha, N}$ values for $T=4 \mathrm{keV}$ as functions of the kinetic energy (left panel) and those for $E_{N}=1 \mathrm{TeV}$ as functions of the cosmic temperature (right panel). Here, $N=n, \bar{n}, p$, and $\bar{p}$, and we have $\eta=6.1 \times 10^{-10}$ and $Y_{p}=0.25$.

hadrodissociation by the antinucleons. On the other hand, by the effects of the interconversion at inelastic scattering, the constraints become weaker by $50 \%-80 \%$. That is because the high-energy protons which can be produced by interconversion from a projectile neutron tend to be stopped more easily than neutrons through electromagnetic interactions inside the thermal plasma.

For comparison, in Fig. 10, we plot $\tilde{\xi}_{\alpha, N}$ using the current method as well as the old method [28], which does not have the interconversion between $n$ and $p$ at inelastic scattering. We can see that the current $\tilde{\xi}_{\alpha, n}$ is reduced to about half of the old one because neutrons are converted to protons during the hadronic shower evolutions. (Notice that protons are stopped more easily than neutrons.) On the other hand, the current $\tilde{\xi}_{\alpha, p}$ is larger than the old one because of the conversion from protons to neutrons.

With $\xi_{A_{i}}\left(T, Y_{p}, \eta\right)$ having been given, the effects of the hadrodissociation are included into the Boltzmann equations which govern the evolutions of the light element abundance; for $A_{i}=n, \mathrm{~T}, \mathrm{D}$, and ${ }^{3} \mathrm{He}$, we use

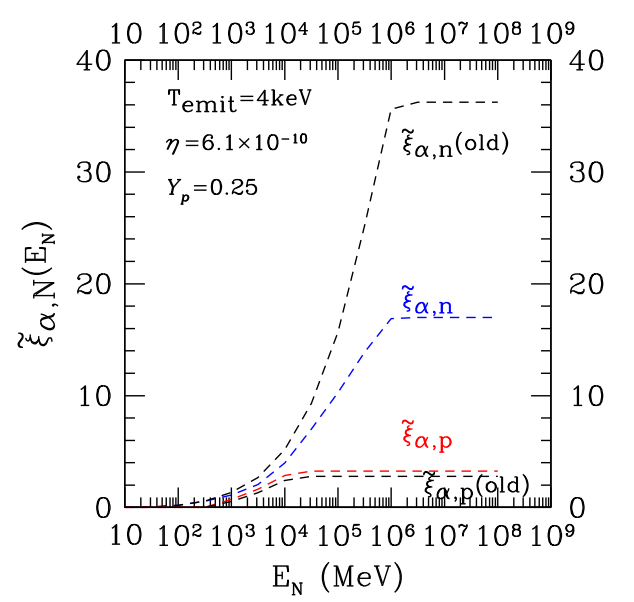

FIG. 10. $\quad \tilde{\xi}_{\alpha, N}$ values for $T=4 \mathrm{keV}$ as functions of the kinetic energy, plotted using the current method (the red and blue lines) as well as the old method, which is without the interconversion between $n$ and $p$ at inelastic scattering (the black lines). Here, we follow the same parameters as those used in the left panel of Fig. 9. 

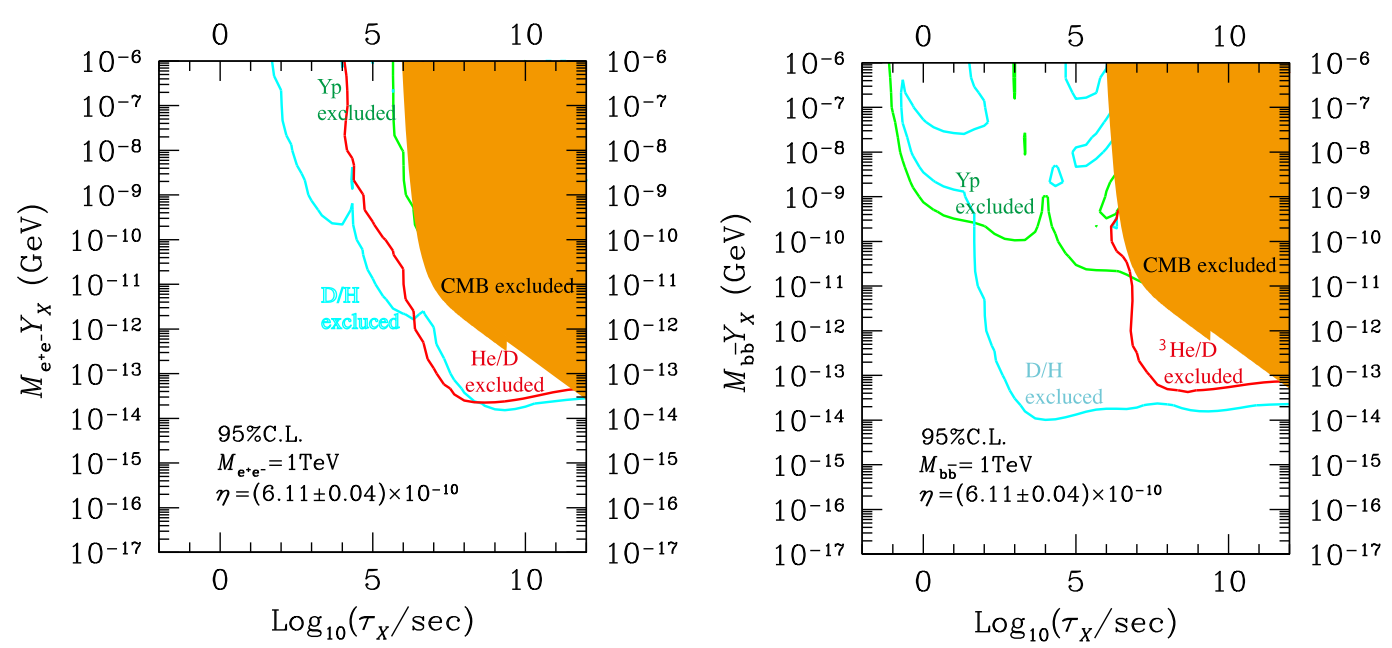

FIG. 11. Constraints on $m_{X} Y_{X}$ vs the $\tau_{X}$ plane, assuming that the main decay modes are $e^{+} e^{-}$(left panel) or $b \bar{b}$ (right panel). The BBN constraints come from ${ }^{4} \mathrm{He}$ (the green lines), $\mathrm{D}$ (the cyan lines), and ${ }^{3} \mathrm{He} / \mathrm{D}$ (the red lines). The orange shaded region is excluded by the CMB spectral distortion.

$$
\frac{d n_{A_{i}}}{d t}=\left[\frac{d n_{A_{i}}}{d t}\right]_{\mathrm{SBBN}}+\left[\frac{d n_{A_{i}}}{d t}\right]_{\text {photodis }}+n_{X} \Gamma_{X} \xi_{A_{i}}
$$

while

$\frac{d n^{{ }^{H} \mathrm{He}}}{d t}=\left[\frac{d n^{{ } \mathrm{He}}}{d t}\right]_{\mathrm{SBBN}}+\left[\frac{d n^{{ } \mathrm{He}}}{d t}\right]_{\text {photodis }}-n_{X} \Gamma_{X} \xi_{\alpha}$

Here, the subscripts SBBN and photodis imply the reaction rates due to the SBBN and photodissociation processes. Notice that the effects of the $p \leftrightarrow n$ interconversion of the background nucleons are included in the SBBN contributions by properly modifying the number densities of the backgrounds $p$ and $n$.

For ${ }^{6} \mathrm{Li},{ }^{7} \mathrm{Li}$, and ${ }^{7} \mathrm{Be}$, we include nonthermal production processes induced by secondary energetic $\mathrm{T},{ }^{3} \mathrm{He}$, and ${ }^{4} \mathrm{He}$. Such energetic nuclei are produced by the hadronic scattering of energetic nucleons off the background $\alpha_{\mathrm{BG}}$, as well as by the electromagnetic processes of energetic photons. For the nonthermal processes induced by hadrons, the effects are parametrized by the following quantity (with $A_{f}={ }^{6} \mathrm{Li},{ }^{7} \mathrm{Li}$, and $\left.{ }^{7} \mathrm{Be}\right)[22-24,28]$ :

$$
\begin{aligned}
\xi_{A_{f}}= & \sum_{A_{i}=\mathrm{T},{ }^{3} \mathrm{He},{ }^{4} \mathrm{He}} \int d E_{A_{i}}^{(\mathrm{in})} f_{A_{i}}\left(E_{A_{i}}^{(\mathrm{in})}\right) \int^{E_{A_{i}}^{(\mathrm{in})}} d E_{A_{i}}\left(\frac{d E_{A_{i}}}{d t}\right)^{-1} \\
& \times n_{\alpha} \sigma_{A_{i}+\alpha_{\mathrm{BG}} \rightarrow A_{f}+\cdots}\left(E_{A_{i}}\right) \beta_{A_{i}} P_{A_{f}}
\end{aligned}
$$

where $f_{A_{i}}$ is the energy distribution of $A_{i}$ produced by the scattering or hadrodissociation processes of $\alpha_{\mathrm{BG}}$, $\left(d E_{A_{i}} / d t\right)^{-1}$ is the energy-loss rate, $\sigma_{A_{i}+\alpha_{\mathrm{BG}} \rightarrow A_{f}+\ldots}$ is the production cross section of $A_{f}, \beta_{A_{i}}$ is the velocity, and $P_{A_{f}}$ is the survival probability of $A_{f}$ after production. (For $A_{f}={ }^{6} \mathrm{Li}, A_{i}=\mathrm{T},{ }^{3} \mathrm{He}$, and ${ }^{4} \mathrm{He}$ contributes, while only
$A_{i}={ }^{4} \mathrm{He}$ is relevant for $A_{f}={ }^{7} \mathrm{Li}$ and ${ }^{7} \mathrm{Be}$.) Then, for $A_{i}={ }^{6} \mathrm{Li},{ }^{7} \mathrm{Li}$, and ${ }^{7} \mathrm{Be}$, the Boltzmann equations are given in the following form:

$$
\begin{aligned}
\frac{d n_{A_{i}}}{d t}= & {\left[\frac{d n_{A_{i}}}{d t}\right]_{\mathrm{SBBN}}+\left[\frac{d n_{A_{i}}}{d t}\right]_{\text {photodis }}+\left[\frac{d n_{A_{i}}}{d t}\right]_{\gamma+\alpha_{\mathrm{BG}} \rightarrow \cdots} } \\
& +n_{X} \Gamma_{X} \xi_{\mathrm{Ai}} .
\end{aligned}
$$

Here, $\left[d n_{A_{i}} / d t\right]_{\gamma+\alpha_{\mathrm{BG}} \rightarrow \cdots}$ denotes the effects of the nonthermal production processes initiated by the photodissociation of $\alpha_{\mathrm{BG}}$ [16], which exists only for $A_{i}={ }^{6} \mathrm{Li}$.

The expansion of the Universe is determined by the Einstein equation; the expansion rate of the Universe is calculated while taking into account the energy density of $X$. In addition, the effect of the entropy production due to the decay of $X$ is included. Thus, the value of $\eta$ is defined as the value after the entropy production. ${ }^{8}$ The initial value of the baryon number density is set to realize the required value of $\eta$. These effects are relevant for the parameter region in which $X$ once dominated the Universe. (As we will see below, however, such a parameter region is mostly excluded by the CMB constraints.)

\section{CONSTRAINTS ON GENERIC DECAYING PARTICLES}

\section{A. Constraints from each light element}

We first investigate the BBN constraints on generic decaying particles. In Fig. 11, we show the constraints on the decaying particles which primarily decay into

\footnotetext{
${ }^{8}$ We consider the case where the same amount of baryons and antibaryons are produced, on average, by the decay. For the case of an asymmetric decay, see, for example, Ref. [49].
} 
$e^{+} e^{-}$or $b \bar{b}$. In the figure, we show the constraints on the combination $m_{X} Y_{X}$ (with $m_{X}$ being the mass of $X$ ) as functions of the lifetime $\tau_{X}$ for the final states $e^{+} e^{-}$ or $b \bar{b}{ }^{9}$

When the decaying particles mainly decay into $e^{+} e^{-}$, the hadronic branching ratio is small. In fact, hadrons are produced through the decay process $X \rightarrow e^{+}+e^{-}+$ $q+\bar{q}$, but the branching ratio for such a process is suppressed by $\sim \mathcal{O}\left((\alpha / 4 \pi)^{2}\right) \sim 10^{-6}$. Thus, most of the constraints are due to radiative decay. At the cosmic time $\tau_{X} \sim 10^{4}-10^{6} \mathrm{sec}, \mathrm{D}$ is destroyed only by energetic photons that are not thermalized by photon-photon processes, so the photodissociation of $\mathrm{D}$ gives a stringent constraint for $\tau_{X} \sim 10^{4}-10^{6} \mathrm{sec}$. At $t \gtrsim 10^{6} \mathrm{sec},{ }^{4} \mathrm{He}$ is also destroyed by the photodissociation processes, which leads to nonthermal production of $\mathrm{D}$ and ${ }^{3} \mathrm{He}$. Thus, the stringent constraints are imposed by overproduction of $\mathrm{D}$ and ${ }^{3} \mathrm{He} .{ }^{10}$ It is seen that the abundance of the $X$ is also constrained for $\tau_{X} \lesssim 10^{4} \mathrm{sec}$, which is due to the hadrodissociation of ${ }^{4} \mathrm{He}$. Since the photons with energy larger than $\mathcal{O}(1) \mathrm{MeV}$ are quickly thermalized at $t \lesssim 10^{4} \mathrm{sec}$, they cannot destroy the light elements. On the other hand, hadrons like protons and neutrons can destroy ${ }^{4} \mathrm{He}$ and produce $\mathrm{D}$ and ${ }^{3} \mathrm{He}$ nonthermally. The resultant constraint due to hadrons is weak because the hadronic branching ratio is small in this case.

In the case where $X$ mainly decays into $b \bar{b}$ [Fig. 11 (right panel)], the stringent constraints come from hadrodissociation of the light elements. The high-energy quarks emitted in the decay induce hadronic showers in which ${ }^{4} \mathrm{He}$ nuclei are destroyed by energetic nucleons. The hadrodissociation of ${ }^{4} \mathrm{He}$ leads to overproduction of $\mathrm{D}$, which gives a particularly stringent constraint for $\tau_{X} \sim 10^{2}-10^{7} \mathrm{sec}$. The photodissociation of ${ }^{4} \mathrm{He}$ also produces $\mathrm{D}$ and gives a stringent constraint for $\tau_{X} \gtrsim 10^{7} \mathrm{sec}$ where the effects of hadrodissociation and photodissociation are roughly comparable. In addition, the nonthermal production of ${ }^{3} \mathrm{He}$ by photodissociation gives a significant constraint when $\tau_{X} \gtrsim 10^{7} \mathrm{sec}$. When $\tau_{X} \lesssim 100 \mathrm{sec}$, the constraint coming from ${ }^{4} \mathrm{He}$ overproduction is most stringent. At the early stage of $\mathrm{BBN}(t \sim 1-100 \mathrm{sec})$, the interconversion of protons and neutrons is the most important process which almost determines the final ${ }^{4} \mathrm{He}$ abundance. The strongly interacting conversion increases $n / p$ from its standard value. As a result, more ${ }^{4} \mathrm{He}$ is produced, from which we obtain the constraint for $\tau_{X} \lesssim 100 \mathrm{sec}$. From the figure, one notices that there appears to be a constraint from $\mathrm{D} / \mathrm{H}$

\footnotetext{
${ }^{9}$ In the figures, in order to indicate the final state, we use the notation $M_{F F^{\prime}}$, which is equal to $m_{X}$, where $F F^{\prime}$ corresponds to the final-state particles of the $X$ decay.

${ }^{10}$ Notice that the present constraints from ${ }^{3} \mathrm{He} / \mathrm{D}$ and $\mathrm{D} / \mathrm{H}$ are almost the same, while in the previous work [28] the constraint from ${ }^{3} \mathrm{He} / \mathrm{D}$ was more severe than that from $\mathrm{D} / \mathrm{H}$. This is because of the recent precise measurement of the abundance of $\mathrm{D} / \mathrm{H}$.
}

for $\tau_{X} \sim 0.1-100 \mathrm{sec}$. The constraint around $m_{X} Y_{X} \sim$ $10^{-9} \mathrm{GeV}$ comes from an overproduction of $\mathrm{D}$ due to a larger $n / p$ value. Since the change of $n / p$ affects the abundance of ${ }^{4} \mathrm{He}$ more significantly, the $\mathrm{D} / \mathrm{H}$ constraint is weaker. The D/H constraint around $m_{X} Y_{X} \sim 10^{-7} \mathrm{GeV}$ calls for caution. In this parameter region, a large $n / p$ value already makes the abundance of ${ }^{4} \mathrm{He}$ much larger than the standard value. The $\mathrm{D}$ abundance is sensitive to the change of ${ }^{4} \mathrm{He}$ abundance because $\mathrm{D}$ is residual after synthesizing ${ }^{4} \mathrm{He}$. The ${ }^{4} \mathrm{He}$ abundance has $\mathcal{O}(10) \%$ theoretical uncertainty in this parameter region, which drastically increases the uncertainty of the D abundance. This makes it difficult to obtain a reliable constraint from D. Since this region is ruled out by the overproduction of ${ }^{4} \mathrm{He}$, this is not an obstacle to obtain constraints on the properties of $X$.

In Fig. 11, the constraints from the CMB are also shown. When the decay of $X$ injects electromagnetic energy into the background plasma, the spectrum of the CMB is distorted. Since photon number changing processes like double Compton scattering are not efficient at $t \gtrsim 10^{7} \mathrm{sec}$, the resultant CMB spectrum deviates from the Planck distribution if $X$ decays at such an epoch [50-52]. The COBE sets a stringent constraint on the spectrum distortion of the CMB [53], from which we obtain the upper bound on the abundance of $X$, as shown in Fig. 11. It is seen that the BBN constraints are more stringent than those from the CMB for both decay modes. ${ }^{11}$ Notice that future observations of the CMB spectrum such as PIXIE will improve the bounds by a factor of 100-500 [55].

\section{B. Constraints on various decay modes}

Now we discuss the constraints for cases with various main decay modes.

We first consider cases where $X$ decays into colored particles. In Fig. 12, we show the combined constraints on the abundance and lifetime of $X$, whose main decay mode is $u \bar{u}, b \bar{b}, t \bar{t}$, or $g g$; in Fig. 12, only the most stringent constraint is shown combining the constraints from $\mathrm{D},{ }^{4} \mathrm{He}$, and ${ }^{3} \mathrm{He} / \mathrm{D}$. For all of the decay modes shown in this figure, the decay products are colored particles and produce hadronic showers whose total energy is roughly equal to $m_{X}$. Therefore, the resultant constraints are similar. As described above, hadrons produced by the decay affect BBN for $\tau_{X} \lesssim 10^{7} \mathrm{sec}$. As a result, constraints from the overproduction of ${ }^{4} \mathrm{He}$ due to the enhancement of $n / p$ are most stringent for $\tau_{X} \lesssim 10^{2} \mathrm{sec}$, while overproduction of D via the hadrodissociation of ${ }^{4} \mathrm{He}$ gives the strongest constraint for $\tau_{X} \gtrsim 10^{2}-10^{7} \mathrm{sec}$. We also show how the constraints depend on $m_{X}$, taking $m_{X}=0.03,0.1,1,10$, 100 , and $1000 \mathrm{TeV}$. Since the number of hadrons produced

\footnotetext{
${ }^{11}$ See also Ref. [54], for a possible mass dependence on this relation.
} 

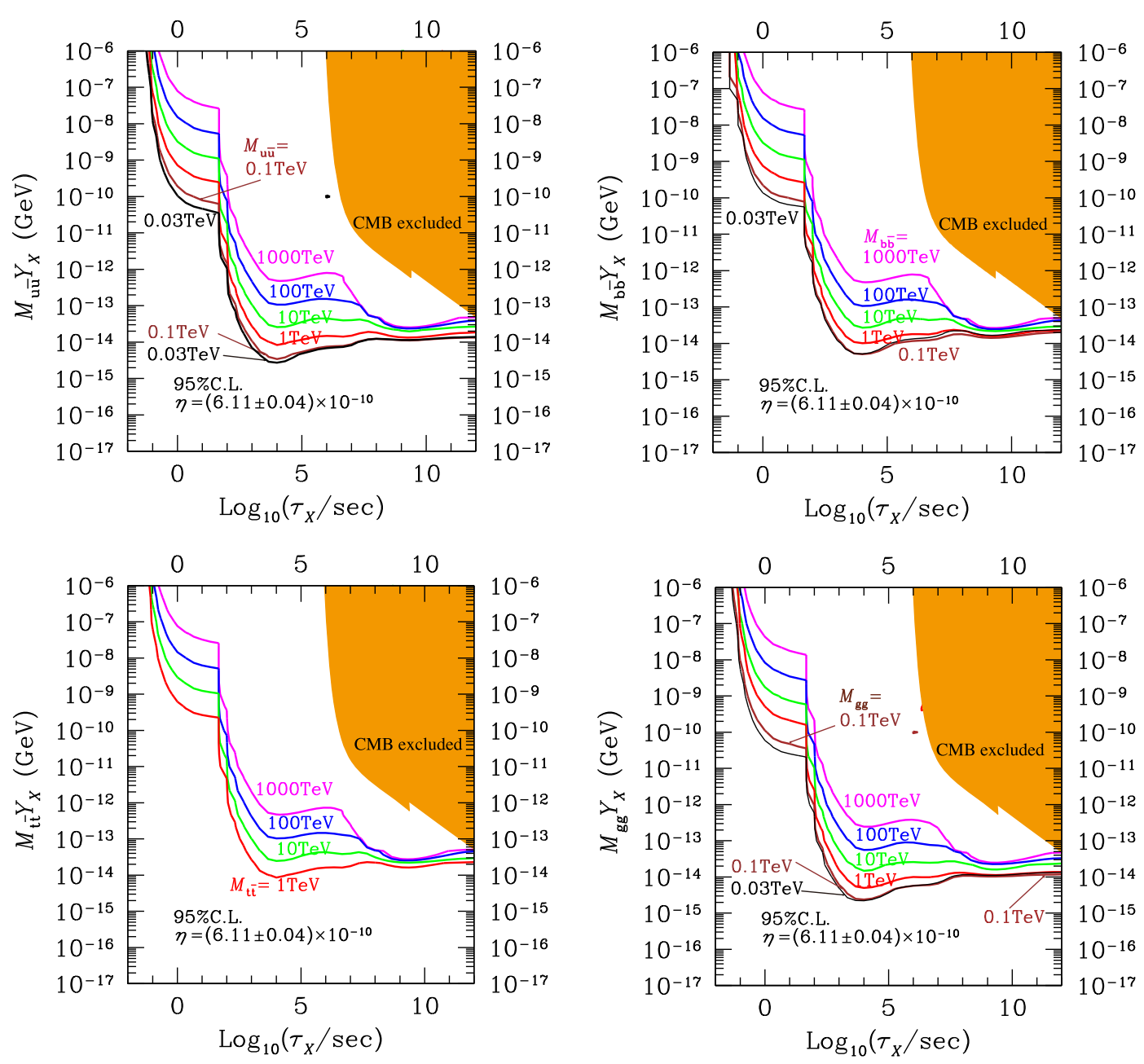

FIG. 12. Constraints on the $m_{X} Y_{X}$ vs $\tau_{X}$ plane assuming that the main decay modes are $u \bar{u}$ (upper left panel), $b \bar{b}$ (upper right panel), $t \bar{t}$ (lower left panel), and $g g$ (lower right panel). The black, red, green, blue, and magenta solid lines denote the BBN constraints for $m_{X}=0.03,0.1,1,10,100$, and $1000 \mathrm{TeV}$, respectively. The orange shaded regions are excluded by the constraint from the $\mathrm{CMB}$ spectral distortion.

through the hadronization process depends on $m_{X}$ as $m_{X}^{\delta}$ with $\delta \sim 0.3$, the constraints on $m_{X} Y_{X}$ from hadrodissociation become weaker as $m_{X}$ increases. On the other hand, the most stringent constraints for $\tau_{X} \gtrsim 10^{7} \mathrm{sec}$ come from the photodissociation of ${ }^{4} \mathrm{He}$, which leads to the overproduction of D and ${ }^{3} \mathrm{He}$. Since the effects of photodissociation are determined by the total energy injection, the constraint depends only on $m_{X} Y_{X}$, as confirmed in Fig. 12.

In Fig. 13, we show the combined constraints on $X$, which decays mainly into $e^{+} e^{-}, \tau^{+} \tau^{-}, \gamma \gamma$, or $W^{+} W^{-}$, taking $m_{X}=0.03,0.1,1,10,100$, and $1000 \mathrm{TeV}$. For the decay into $e^{+} e^{-}$, as described in Sec. IVA, the constraints are determined by the photodissociation effect for $\tau_{X} \gtrsim$ $10^{4} \mathrm{sec}$ (the destruction of $\mathrm{D}$ for $\tau_{X} \sim 10^{4}-10^{6} \mathrm{sec}$ and the overproduction of ${ }^{3} \mathrm{He}$ for $\tau_{X} \gtrsim 10^{6} \mathrm{sec}$ ). Because the branching ratio into $q \bar{q}$ is small, the effect of hadronic decay is significant only when $\tau_{X} \lesssim 10^{4} \mathrm{sec}$, where photodissociation does not take place. The constraints for the case in which $X$ decays mainly into $\tau^{+} \tau^{-}$are also shown. Since the branching ratio into $q \bar{q}$ is as small as the $e^{+} e^{-}$case, the constraints where $\tau_{X} \gtrsim 10^{4} \mathrm{sec}$ are almost the same as Fig. 13 (upper left panel); the constraints are slightly weaker because a certain amount of the energy is carried away by neutrinos. The effect of hadrodissociation is seen when $\tau_{X} \lesssim 10^{4} \mathrm{sec}$, which is similar to the $e^{+} e^{-}$ decay. The constraints from the change of $n / p$ are seen when $\tau_{X} \lesssim 10^{2}$ sec, which is due to the mesons produced by the $\tau$ decay. The constraints on the decay mode $\gamma \gamma$ are shown in Fig. 13 (lower left panel). For $\tau_{X} \gtrsim 10^{6} \mathrm{sec}$, photodissociation is important and the constraint is similar to that for $e^{+} e^{-}$decay. However, compared to the $e^{+} e^{-}$ case, the $q \bar{q}$ production rate at the decay is relatively large, $\sim \mathcal{O}(\alpha / 4 \pi) \sim 10^{-4}-10^{-3}$. Therefore, the constraints from the hadronic processes are more important than those from photodissociation when $\tau_{X} \lesssim 10^{6}$ sec. In Fig. 13 (lower 

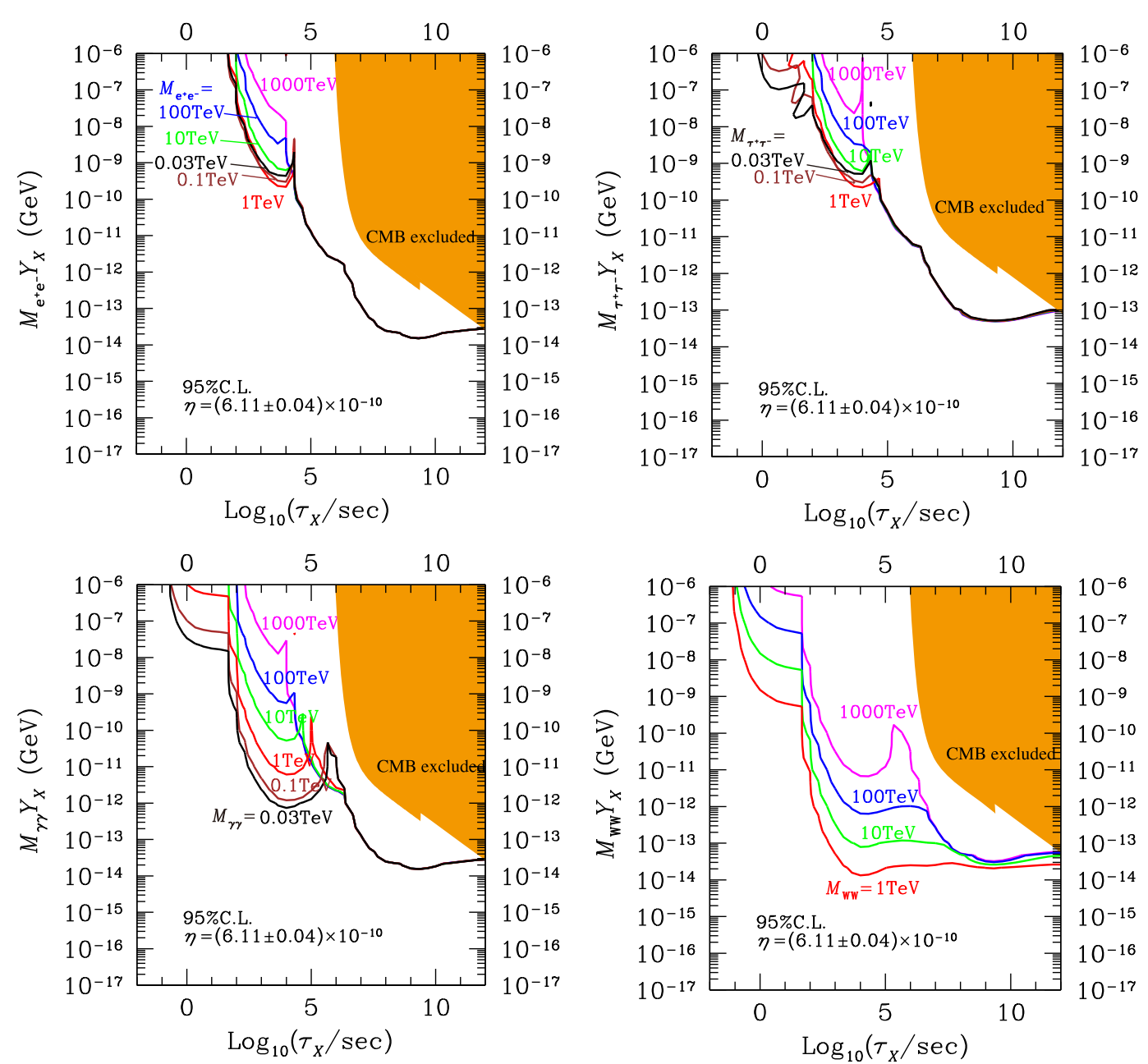

FIG. 13. Constraints on the $m_{X} Y_{X}$ vs $\tau_{X}$ plane assuming that the main decay modes are $e^{+} e^{-}$(upper left panel), $\tau^{+} \tau^{-}$(upper right panel), $\gamma \gamma$ (lower left panel), and $W^{+} W^{-}$(lower right panel). The black, red, green, blue, and magenta solid lines denote the BBN constraints for $m_{X}=0.03,0.1,1,10,100$, and $1000 \mathrm{TeV}$, respectively. The orange shaded regions are excluded by the constraint from the CMB spectral distortion.

right panel), we show the constraints on the decay into $W^{+} W^{-}$. Since $W$ bosons further decay into hadrons with a branching ratio of about 0.67 , effects of the hadronic decay are important, and hence the resultant constraints look similar to those on the $q \bar{q}$ decay modes.

Thus far, we have adopted Eq. (2.4) as the observational constraint of ${ }^{4} \mathrm{He}$. Now let us see how the constraints change if we adopt the other constraint (2.3). In Fig. 14, the $\mathrm{BBN}$ constraints from $\mathrm{D},{ }^{3} \mathrm{He} / \mathrm{D}$, and ${ }^{4} \mathrm{He}$ are shown, assuming that the main decay mode is $b \bar{b}$. As mentioned in Sec. II, the ${ }^{4} \mathrm{He}$ abundance (2.3) estimated by Izotov et al. [38] is significantly larger than that obtained by Aver et al. [39] given in Eq. (2.4), and it is not consistent with SBBN if we use the baryon-to-photon ratio determined by Planck. Their observation becomes consistent with BBN if the abundance of ${ }^{4} \mathrm{He}$ is increased by the decay of $X$. When $X$ mainly decays into $b \bar{b}$, there appears to be a region consistent with Eq. (2.3), as well as with other light element abundance, i.e., $\tau_{X} \sim 10^{-1}-10^{4} \mathrm{sec}$ and $m_{X} Y_{X} \sim 10^{-10}-10^{-6} \mathrm{GeV}$. This is due to the effect that the decay of $X$ with such lifetime induces the $p \leftrightarrow n$ conversion, resulting in an increase in the abundance of ${ }^{4} \mathrm{He}$. If the ${ }^{4} \mathrm{He}$ abundance (2.3) is confirmed, this may suggest the existence of a long-lived hadronically decaying particle which solves the discrepancy between SBBN and Eq. (2.3). On the other hand, if the hadronic branching ratio is much smaller, like the case that the main decay mode is $e^{+} e^{-}$, there is no region consistent with Eq. (2.3) together with observational constraints on $\mathrm{D}$ and ${ }^{3} \mathrm{He} / \mathrm{D}$.

Before closing this section, we comment on the effects of the decaying particles on the abundance of ${ }^{7} \mathrm{Li}$ and ${ }^{6} \mathrm{Li}$, although we do not use them to derive the constraints on the decaying particles. In Fig. 15, the abundance of ${ }^{7} \mathrm{Li}$ and ${ }^{6} \mathrm{Li}$ is shown, assuming that the main decay mode is $b \bar{b}$ or 


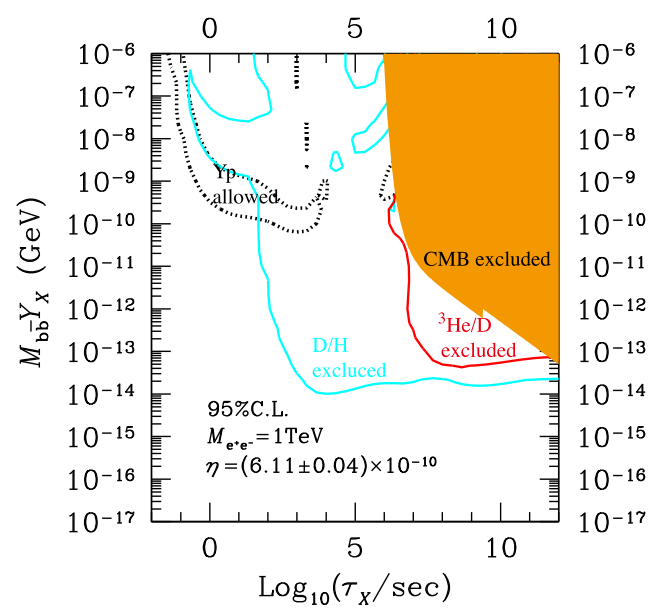

FIG. 14. Constraints on $m_{X} Y_{X}$ vs $\tau_{X}$ plane, assuming that $m_{X}=$ $1 \mathrm{TeV}$ and that the main decay mode is $b \bar{b}$. The solid cyan, solid red, and dashed lines denote the BBN constraints from $\mathrm{D},{ }^{3} \mathrm{He} / \mathrm{D}$, and ${ }^{4} \mathrm{He}$, respectively. Here, we use Eq. (2.3) as the observational constraint on $Y_{p}$. The orange shaded region is excluded by the CMB spectral distortion.
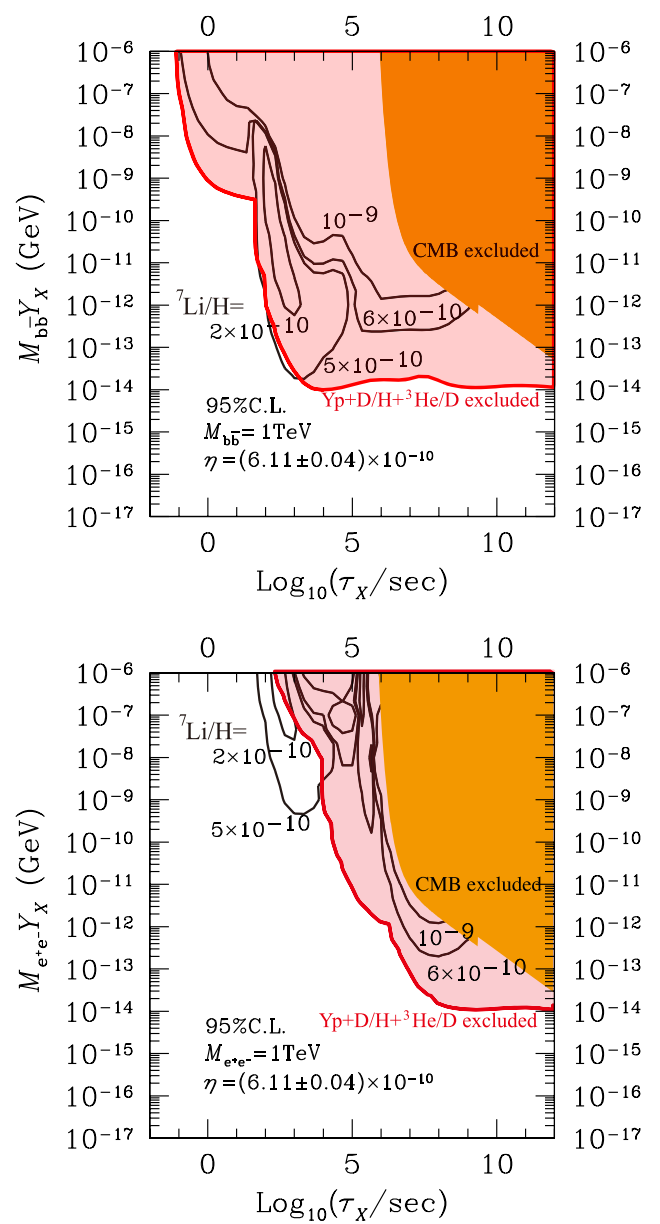

$e^{+} e^{-}$. Notably, for $\tau_{X} \sim 10^{2}-10^{3} \mathrm{sec}$, primordial ${ }^{7} \mathrm{Li}$ abundance is reduced by nonthermal neutrons; ${ }^{7} \mathrm{Be}$, which is one of the origins of primordial ${ }^{7} \mathrm{Li}$, can be converted to ${ }^{7} \mathrm{Li}$ by nonthermal neutrons as ${ }^{7} \mathrm{Be}+n \rightarrow{ }^{7} \mathrm{Li}+p .{ }^{7} \mathrm{Li}$ is then destroyed by the $\mathrm{SBBN}$ reaction ${ }^{7} \mathrm{Li}+p \rightarrow$ ${ }^{4} \mathrm{He}+{ }^{4} \mathrm{He}$. Such effects may dominate over the nonthermal production of ${ }^{7} \mathrm{Li}$ due to energetic $\mathrm{T},{ }^{3} \mathrm{He}$, and ${ }^{4} \mathrm{He}$ from the photodissociation and scattering of ${ }^{4} \mathrm{He}$, resulting in a net decrease of the ${ }^{7} \mathrm{Li}$ abundance. For the case involving the $b \bar{b}$ mode, the constraints from $\mathrm{D} / \mathrm{H},{ }^{3} \mathrm{He} / \mathrm{D}$, and ${ }^{4} \mathrm{He}$ exclude the parameter region where the ${ }^{7} \mathrm{Li}$ abundance significantly decreases. On the other hand, ${ }^{7} \mathrm{Li}$ abundance can be reduced for the main decay mode of $e^{+} e^{-}$without conflicting with other constraints if $\tau_{X} \sim \mathcal{O}(100) \mathrm{sec}$ and $m_{X} Y_{X} \sim 10^{-7} \mathrm{GeV}$. This might provide a solution to the ${ }^{7} \mathrm{Li}$ problem in SBBN.

In Fig. 15, it is seen that nonthermal production of ${ }^{6} \mathrm{Li}$ due to the decaying particles is significant for $\tau_{X} \gtrsim 10^{3}$ sec. Taking into account the constraints from the other light elements, ${ }^{6} \mathrm{Li} /{ }^{7} \mathrm{Li}$ can be as large as $10^{-2}$ for
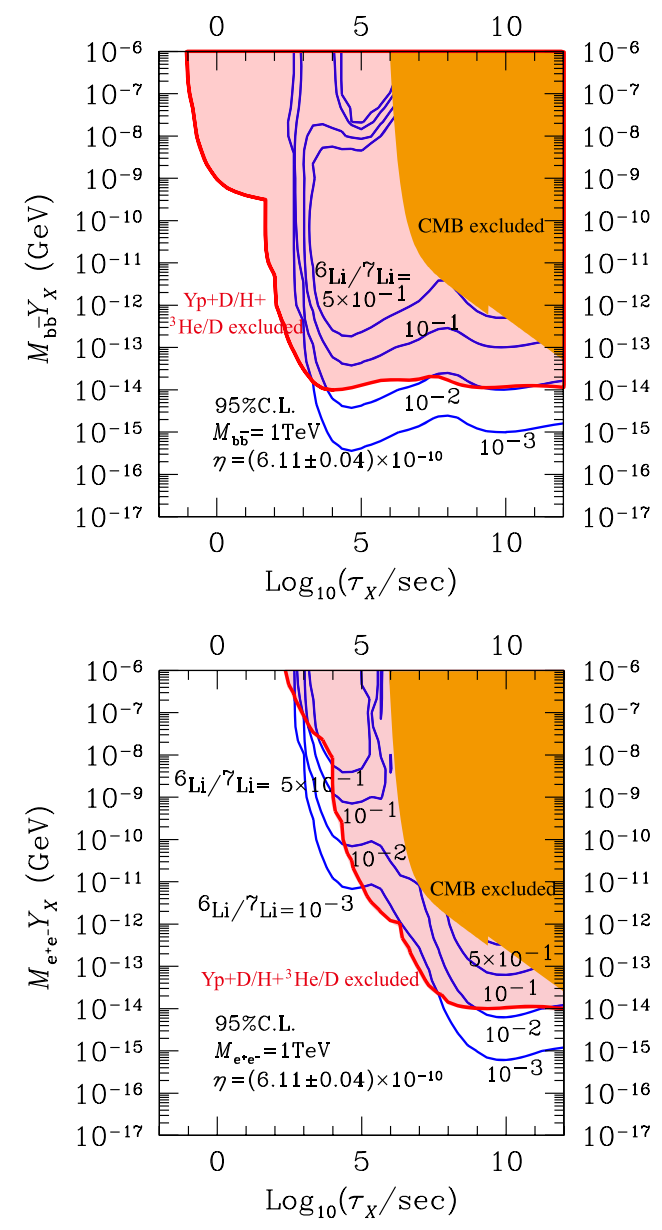

FIG. 15. Abundance of ${ }^{7} \mathrm{Li} / \mathrm{H}$ (left panels) and ${ }^{6} \mathrm{Li} /{ }^{7} \mathrm{Li}$ (right panels) for decay particles with a mass of 1 TeV which decay mainly into $b \bar{b}$ (upper panels) and $e^{+} e^{-}$(lower panels). The constraints from other light elements and CMB are shown in the pink shaded region (surrounded by the red solid line) and the orange shaded region, respectively. 
the $b \bar{b}$ decay mode, while it can be $\mathcal{O}(0.1)$ for the $e^{+} e^{-}$ mode. On the contrary, ${ }^{6} \mathrm{Li}$ is hardly produced in SBBN (i.e., ${ }^{6} \mathrm{Li} /{ }^{7} \mathrm{Li} \lesssim 10^{-4}$ ). Thus, if a significant amount of ${ }^{6} \mathrm{Li} /{ }^{7} \mathrm{Li}$ is observed in low-metal stars, it would provide evidence for the existence of decaying particles in the early Universe. Particularly for the $e^{+} e^{-}$mode, it is remarkable that ${ }^{6} \mathrm{Li} /{ }^{7} \mathrm{Li}$ is significantly large when we simultaneous solve the ${ }^{7} \mathrm{Li}$ problem at around $\tau_{X} \sim 10^{3} \mathrm{sec}$ and $m_{X} Y_{X} \sim 10^{-7.5} \mathrm{GeV}$.

\section{GRAVITINO}

One of the important candidates for long-lived particles is the gravitino in SUSY models. A gravitino is the superpartner of a graviton, and it interacts very weakly because its interaction is suppressed by inverse powers of the (reduced) Planck scale $M_{\mathrm{Pl}} \simeq 2.4 \times 10^{18} \mathrm{GeV}$. Because of the weakness of its interaction, the lifetime of a gravitino may become so long that its decay products affect the light element abundance (if the gravitino is unstable). Assuming $R$-parity conservation, a gravitino becomes unstable if it is not the lightest superparticle (LSP). Even when a gravitino is the LSP, the nextto-lightest superparticle (NLSP) decays into a gravitino with a very long lifetime, and hence the BBN constraints on the properties of the NLSP are derived; see, e.g., Refs. [31,56]. Here, we pay particular attention to the former case where the gravitino is unstable because, in such a case, we can obtain an upper bound on the reheating temperature after inflation in order not to overproduce gravitino. Applying the analysis of the nonstandard BBN processes discussed in earlier, we study the effects of gravitino decay on light element abundance and derive the upper bound on the reheating temperature.

The primordial abundance of a gravitino is sensitive to the reheating temperature after inflation, ${ }^{12}$ which we define as

$$
T_{\mathrm{R}} \equiv\left(\frac{10}{g_{*}\left(T_{\mathrm{R}}\right) \pi^{2}} M_{\mathrm{Pl}}^{2} \Gamma_{\text {inf }}^{2}\right)^{1 / 4}
$$

with $\Gamma_{\text {inf }}$ being the decay rate of the inflaton and $g_{*}\left(T_{\mathrm{R}}\right)$ being the effective number of the massless degrees of freedom at the time of reheating. In our paper, we use the value suggested by the minimal SUSY standard model $(\mathrm{MSSM}), g_{*}\left(T_{\mathrm{R}}\right)=228.75$.

A gravitino is produced via the scattering processes of MSSM particles in the thermal bath. The Boltzmann equation for the number density of a gravitino (denoted as $n_{3 / 2}$ ) is given by

\footnotetext{
${ }^{12} \mathrm{~A}$ gravitino may be also produced nonthermally by the decay of the moduli fields or inflaton field [57-59]. We do not consider such contributions in our work to derive a conservative bound on the reheating temperature after inflation.
}

$$
\frac{d n_{3 / 2}}{d t}+3 H n_{3 / 2}=C_{3 / 2}
$$

where $C_{3 / 2}$ is the thermally averaged collision term. The most precise calculation of the collision term includes hard thermal loop resummation to avoid infrared singularity [60-62], and $C_{3 / 2}$ is parametrized as

$$
C_{3 / 2}=\sum_{i=1}^{3} \frac{3 \zeta(3) T^{6}}{16 \pi^{3} M_{\mathrm{Pl}}^{2}}\left(1+\frac{M_{i}^{2}(T)}{3 m_{3 / 2}^{2}}\right) c_{i} g_{i}^{2} \ln \left(\frac{k_{i}}{g_{i}}\right),
$$

where $i=1,2$, and 3 correspond to the gauge groups $U(1)_{Y}, S U(2)_{L}$, and $S U(3)_{C}$, respectively. Here, $M_{i}(T)$ represents the gaugino mass parameters at the renormalization scale $Q=T$, and the $g_{i}$ values are the gauge coupling constants. In addition, $c_{i}$ and $k_{i}$ are numerical constants. References [60,61] give $\left(c_{1}, c_{2}, c_{3}\right)=(11,27,72)$ and $\left(k_{1}, k_{2}, k_{3}\right)=(1.266,1.312,1.271)$, while the coefficients from Ref. [62] are $\left(c_{1}, c_{2}, c_{3}\right)=(9.90,20.77,43.34)$ and $\left(k_{1}, k_{2}, k_{3}\right)=(1.469,2.071,3.041) .{ }^{13}$

As one can see, the collision term is typically $C_{3 / 2} \sim$ $T^{6} / M_{\mathrm{Pl}}^{2}$ (as long as $T$ is higher than the masses of MSSM particles). Then the yield variable of the gravitino, which is defined as $Y_{3 / 2}$ hereafter, is approximately proportional to the reheating temperature for the parameter region of our interest. Consequently, the BBN constraints on the gravitino abundance can be converted to the upper bound on the reheating temperature $T_{\mathrm{R}}$.

We numerically solve the Boltzmann equation (5.2) (as well as the evolution equation of the Universe based on the Einstein equation) to accurately calculate the primordial abundance of gravitino. Assuming the MSSM particle content up to the grand unified theory (GUT) scale, the gravitino abundance can be well fit by the following formula:

$$
\begin{aligned}
Y_{3 / 2} \simeq & y_{0} T_{\mathrm{R}}^{(8)}\left[1+\delta_{0}^{(1)} \ln T_{\mathrm{R}}^{(8)}+\delta_{0}^{(2)} \ln ^{2} T_{\mathrm{R}}^{(8)}\right] \\
& +\sum_{i=1}^{3} y_{i} T_{\mathrm{R}}^{(8)}\left[1+\delta_{i}^{(1)} \ln T_{\mathrm{R}}^{(8)}+\delta_{i}^{(2)} \ln ^{2} T_{\mathrm{R}}^{(8)}\right] \\
& \times\left(\frac{M_{i}^{(\mathrm{GUT})}}{m_{3 / 2}}\right)^{2},
\end{aligned}
$$

where $T_{\mathrm{R}}^{(8)} \equiv T_{\mathrm{R}} / 10^{8} \mathrm{GeV}$ and $M_{i}^{(\mathrm{GUT})}$ are the gaugino masses at the GUT scale which is taken to be $2 \times 10^{16} \mathrm{GeV}$ in our analysis. Numerical constants in the above fitting formula based on Refs. [60,61] and those for Ref. [62] are summarized in Tables I and II, respectively. The primordial abundance of gravitino based on Refs. [60,61] is smaller than that based on Ref. [62]. Thus, we perform our

\footnotetext{
${ }^{13}$ For the latter, we use the coefficients given in Ref. [63], which is based on Ref. [62].
} 
TABLE I. Numerical constants for the formula (5.4), which gives a fitting formula for the primordial gravitino abundance, based on Refs. [60,61].

\begin{tabular}{lcccc}
\hline \hline & $i=0$ & $i=1$ & $i=2$ & $i=3$ \\
\hline$y_{i}$ & $2.3 \times 10^{-14}$ & $1.3 \times 10^{-16}$ & $1.6 \times 10^{-15}$ & $1.3 \times 10^{-14}$ \\
$\delta_{i}^{(1)}$ & 0.015 & 0.055 & 0.013 & -0.042 \\
$\delta_{i}^{(2)}$ & -0.0005 & 0.0081 & 0.0001 & 0.0006 \\
\hline \hline
\end{tabular}

TABLE II. Numerical constants for the formula (5.4) based on Ref. [62].

\begin{tabular}{lcccc}
\hline \hline & $i=0$ & $i=1$ & $i=2$ & $i=3$ \\
\hline$y_{i}$ & $4.4 \times 10^{-14}$ & $1.5 \times 10^{-16}$ & $2.1 \times 10^{-15}$ & $3.2 \times 10^{-14}$ \\
$\delta_{i}^{(1)}$ & -0.014 & 0.066 & 0.015 & -0.089 \\
$\delta_{i}^{(2)}$ & 0.0054 & 0.0042 & 0.0002 & 0.0031 \\
\hline \hline
\end{tabular}

numerical analysis with the former set of the coefficients in order to derive a conservative constraint. The bound based on Ref. [62] can be obtained by translating the bound on $T_{\mathrm{R}}$ to that on the primordial abundance $Y_{3 / 2}$ using, for e.g., Eq. (5.4); the bound on $T_{\mathrm{R}}$ based on Ref. [62] is, at most, 2 to 3 times more stringent than that based on Refs. [60,61].

Because the interaction of gravitino is governed by the SUSY, the partial decay rates of gravitino are determined once the mass spectrum of the MSSM particles are known. For a precise calculation of the upper bound on the reheating temperature, we fix the mass spectrum of the MSSM particles and calculate the decay widths of the gravitinos. We consider several sample points; the mass spectrum of each sample point is summarized in Table III. Sample point 1 is based on the so-called constrained minimal SUSY standard model (CMSSM) [64] parametrized by the universal scalar mass $m_{0}$, the unified gaugino mass $M_{1 / 2}$, the universal trilinear coupling constants $A_{0}$ (with respect to the corresponding Yukawa coupling constants), $\tan \beta$ (which is the ratio of the vacuum expectation values of up- and down-type Higgs bosons), and the sign of the SUSY invariant Higgs mass parameter $\mu$. For sample point 1 , we take $m_{0}=433 \mathrm{GeV}, M_{1 / 2}=970 \mathrm{GeV}$, $A_{0}=-3020 \mathrm{GeV}, \tan \beta=14$, and $\mu>0$. As one can see, for sample point 1 , all of the MSSM particles are lighter than $\sim 2.5 \mathrm{TeV}$, and the discovery of some of the MSSM particles is expected in a future LHC experiment. Sample point 2 is also based on the CMSSM, with the underlying parameters of $m_{0}=5000 \mathrm{GeV}, M_{1 / 2}=700 \mathrm{GeV}$, $A_{0}=-8000 \mathrm{GeV}, \tan \beta=10$, and $\mu>0$. At this point, the $\mu$ parameter is relatively small, and the Higgsino-like neutralino becomes the LSP. In sample points 3 and 4, we consider the case where the sfermion masses are above
$100 \mathrm{TeV}$, while gaugino masses are near the TeV scale. ${ }^{14}$ Such a mass spectrum is motivated in the so-called pure gravity mediation model [65-67], in which scalar masses originate from a direct Kähler interaction between the SUSY breaking field and the MSSM chiral multiplet, while the gaugino masses are from the effect of anomaly mediation $[68,69]$. Then the gaugino masses are given in the following form:

$$
\begin{gathered}
M_{1}=\frac{g_{1}^{2}}{16 \pi^{2}}\left(11 F_{\Phi}+L\right), \\
M_{2}=\frac{g_{2}^{2}}{16 \pi^{2}}\left(F_{\Phi}+L\right), \\
M_{3}=\frac{g_{3}^{2}}{16 \pi^{2}}\left(-3 F_{\Phi}\right),
\end{gathered}
$$

where $F_{\Phi}$ is the expectation value of the compensator multiplet, ${ }^{15}$ while $L$ parametrizes the effect of the HiggsHiggsino loop on the gaugino masses. In a large class of models, $L$ is on the order of $F_{\Phi}$ for the scale below the masses of heavy Higgses and Higgsinos. The gaugino masses for sample points 3 and 4 are obtained by adopting $\left(F_{\Phi}, L\right)=(131 \mathrm{TeV}, 218 \mathrm{TeV})$, and $(82 \mathrm{TeV}, 87 \mathrm{TeV})$, respectively. Notice that, above the mass scale of the heavy Higgs and Higgsino, the gaugino mass parameters are obtained with Eqs. (5.5)-(5.7), taking $L \rightarrow 0$. For the calculation of the primordial abundance of gravitino, we take this effect into account, and the thermally averaged gravitino production cross sections for points 3 and 4 are evaluated by taking a vanishing $L$ value.

With the fixed mass spectrum of the MSSM particles, we vary the gravitino mass and derive the upper bound on the reheating temperature as a function of the gravitino mass. In our calculation, all of the two-body tree-level decay processes of gravitinos are taken into account. We also include three-body decay processes $\tilde{G} \rightarrow \tilde{\chi}_{0} q \bar{q}$ (with $\tilde{G}, \tilde{\chi}_{0}$, and $q$ denoting the gravitino, the lightest neutralino, and the $u, d, s, c$, or $b$ quark, respectively) if the mass splitting between the gravitino and the lightest neutralino is less than the $Z$-boson mass. This is because those three-body decay processes can make a substantial contribution to hadronic emissions from gravitino decays to the lightest neutralino when the two-body decay process

\footnotetext{
${ }^{14}$ Equation (5.4) is applicable only when the mass scale of the MSSM particles is lower than the reheating temperature. Although this condition is not satisfied when $T_{R} \lesssim 10^{5} \mathrm{GeV}$ for sample points 3 and 4, we use Eq. (5.4) throughout our analysis. As we will see below, the upper bound on $T_{R}$ is found to be at least $\sim 10^{5} \mathrm{GeV}$, and our approximation is marginally acceptable for the reheating temperature of our interest.

${ }^{15}$ In the model where the vacuum expectation value of the SUSY breaking field is much smaller than the Planck scale, $F_{\Phi}=m_{3 / 2}$. In this analysis, however, we consider a more general framework and treat $F_{\Phi}$ as a free parameter.
} 
TABLE III. The mass spectrum of the MSSM particles as well as the gaugino masses at the GUT scale for the sample points adopted in our analysis. Here, $m_{\tilde{u}_{R, i}}$ and $m_{\tilde{u}_{L, i}}\left(m_{\tilde{d}_{R, i}}\right.$ and $\left.m_{\tilde{d}_{L, i}}\right)$ are masses of right- and left-handed sups (s-downs) in the $i$ th generation, respectively, while $m_{\tilde{e}_{R, i}}$ and $m_{\tilde{e}_{L, i}}\left(m_{\tilde{\nu}_{L, i}}\right)$ are masses of right- and left-handed charged sleptons (sneutrinos) in the $i$ th generation, respectively. In addition, $m_{\tilde{t}_{1}}, m_{\tilde{b}_{1}}$, and $m_{\tilde{\tau}_{1}}\left(m_{\tilde{t}_{2}}, m_{\tilde{b}_{2}}\right.$, and $\left.m_{\tilde{\tau}_{2}}\right)$ are lighter (heavier) stop, sbottom, and stau masses, respectively, while $m_{\tilde{\nu}_{\tau_{L}}}$ is the tau-sneutrino mass. Furthermore, $m_{\chi_{i}^{0}}, m_{\chi_{i}^{ \pm}}$, and $m_{\tilde{g}}$ are neutralino, chargino, and gluino masses, respectively, while $m_{h}$ and $m_{A}$ are the masses of the lightest Higgs boson and the $C P$-odd Higgs boson, respectively. All of the mass parameters are given in units of $\mathrm{GeV}$.

\begin{tabular}{|c|c|c|c|c|}
\hline & Point 1 & Point 2 & Point 3 & Point 4 \\
\hline$m_{\tilde{u}_{R, 1,2}}$ & 1907 & 5242 & $\sim 1 \times 10^{5}$ & $\sim 1 \times 10^{5}$ \\
\hline$m_{\tilde{d}_{R, 1,2}}$ & 1898 & 5054 & $\sim 1 \times 10^{5}$ & $\sim 1 \times 10^{5}$ \\
\hline$m_{\tilde{u}_{L, 1,2}}\left(m_{\tilde{d}_{L, 1,2}}\right)$ & $1980(1982)$ & $5082(5083)$ & $\sim 1 \times 10^{5}$ & $\sim 1 \times 10^{5}$ \\
\hline$m_{\tilde{e}_{R, 1,2}}$ & 562 & 4801 & $\sim 1 \times 10^{5}$ & $\sim 1 \times 10^{5}$ \\
\hline$m_{\tilde{e}_{L, 1,2}}\left(m_{\tilde{\nu}_{L, 1,2}}\right)$ & 771 (767) & $5093(5092)$ & $\sim 1 \times 10^{5}$ & $\sim 1 \times 10^{5}$ \\
\hline$m_{\tilde{t}_{1}}$ & 977 & 1455 & $\sim 1 \times 10^{5}$ & $\sim 1 \times 10^{5}$ \\
\hline$m_{\tilde{t}_{2}}$ & 1635 & 3603 & $\sim 1 \times 10^{5}$ & $\sim 1 \times 10^{5}$ \\
\hline$m_{\tilde{b}_{1}}$ & 1608 & 3607 & $\sim 1 \times 10^{5}$ & $\sim 1 \times 10^{5}$ \\
\hline$m_{\tilde{b}_{2}}$ & 1843 & 4990 & $\sim 1 \times 10^{5}$ & $\sim 1 \times 10^{5}$ \\
\hline$m_{\tilde{\tau}_{1}}$ & 417 & 4735 & $\sim 1 \times 10^{5}$ & $\sim 1 \times 10^{5}$ \\
\hline$m_{\tilde{\tau}_{2}}$ & 732 & 5062 & $\sim 1 \times 10^{5}$ & $\sim 1 \times 10^{5}$ \\
\hline$m_{\tilde{\nu}_{\tau_{L}}}$ & 723 & 5061 & $\sim 1 \times 10^{5}$ & $\sim 1 \times 10^{5}$ \\
\hline$m_{\chi_{1}^{0}}$ & 417 & 187 & 1000 & 500 \\
\hline$m_{\chi_{2}^{0}}$ & 791 & -209 & 1470 & 880 \\
\hline$m_{\chi_{3}^{0}}$ & -1836 & 321 & $\sim 1 \times 10^{5}$ & $\sim 1 \times 10^{5}$ \\
\hline$m_{\chi_{4}^{0}}$ & 1838 & 618 & $\sim 1 \times 10^{5}$ & $\sim 1 \times 10^{5}$ \\
\hline$m_{\chi_{1}^{ \pm}}$ & 791 & 199 & 1000 & 500 \\
\hline$m_{\chi_{2}^{ \pm}}$ & 1839 & 618 & $\sim 1 \times 10^{5}$ & $\sim 1 \times 10^{5}$ \\
\hline$m_{\tilde{g}}$ & 2131 & 1817 & 3000 & 2000 \\
\hline$m_{h}$ & 124 & 126 & 125 & 125 \\
\hline$m_{A}$ & 1906 & 1000 & $\sim 1 \times 10^{5}$ & $\sim 1 \times 10^{5}$ \\
\hline$M_{1,2,3}^{(\mathrm{GUT})}$ & 970 & 700 & $(2583,376,-1137)$ & $(1619,236,-716)$ \\
\hline
\end{tabular}

$\tilde{G} \rightarrow \tilde{\chi}_{1}^{0} Z$ is kinematically forbidden. For sample points 3 and 4 , the lightest chargino $\left(\tilde{\chi}_{1}^{ \pm}\right)$has a degenerate mass with the lightest neutralino and behaves as the LSP. We therefore include the off-shell $W^{ \pm}$induced three-body decay processes, $\tilde{G} \rightarrow \tilde{\chi}_{1}^{ \pm} q \bar{q}^{\prime}$, as well as in our calculation if $m_{3 / 2}-m_{\tilde{\chi}_{1}^{ \pm}}<m_{W}$.

The partial decay rates of gravitino are calculated by using the MadGraph5_aMC@NLO v2.1 package [70] with gravitino interactions being implemented via FeynRules v2.3 [71-74]. Subsequent decay and the hadronization processes of the decay products of gravitino are simulated by using PYTHIA8.2 package [42]. For a given reheating temperature, the primordial abundance of gravitino is calculated by numerically solving Eq. (5.2). For the thermally averaged gravitino production cross section, we adopt the results of Refs. $[60,61]$.

Following the procedure discussed in the previous sections, we calculate the light element abundance, taking into account the effects of the decay products of the gravitino. The constraints on the gravitino abundance are then translated to the upper bound on the reheating temperature. The upper bound on $T_{\mathrm{R}}$ for the sample points 1, 2, 3, and 4 are shown in Fig. 16. As one can see, a severe upper bound of $\sim 10^{5}-10^{6} \mathrm{GeV}$ is obtained from the overproduction of $\mathrm{D}$ for a relatively light gravitino mass (i.e., for $m_{3 / 2} \lesssim$ a few $\mathrm{TeV}$ ). This is because, when a gravitino is lighter than a few $\mathrm{TeV}$, the lifetime of the gravitino is longer than $\sim 10^{3} \mathrm{sec}$ so that the constraint from $\mathrm{D}$ is significant, as discussed in the previous section. On the contrary, for a heavier gravitino mass (i.e., for $m_{3 / 2} \gtrsim 10 \mathrm{TeV}$ ), for which the lifetime of the gravitino becomes shorter than $\sim 10^{2} \mathrm{sec}$, the most stringent constraint comes from the overproduction of ${ }^{4} \mathrm{He}$ due to the $p \leftrightarrow n$ conversion.

We also comment on the constraints based on the ${ }^{4} \mathrm{He}$ abundance given in Eq. (2.3), which is not consistent with the SBBN prediction. As mentioned in the previous section, with a hadronically decaying long-lived particle, 

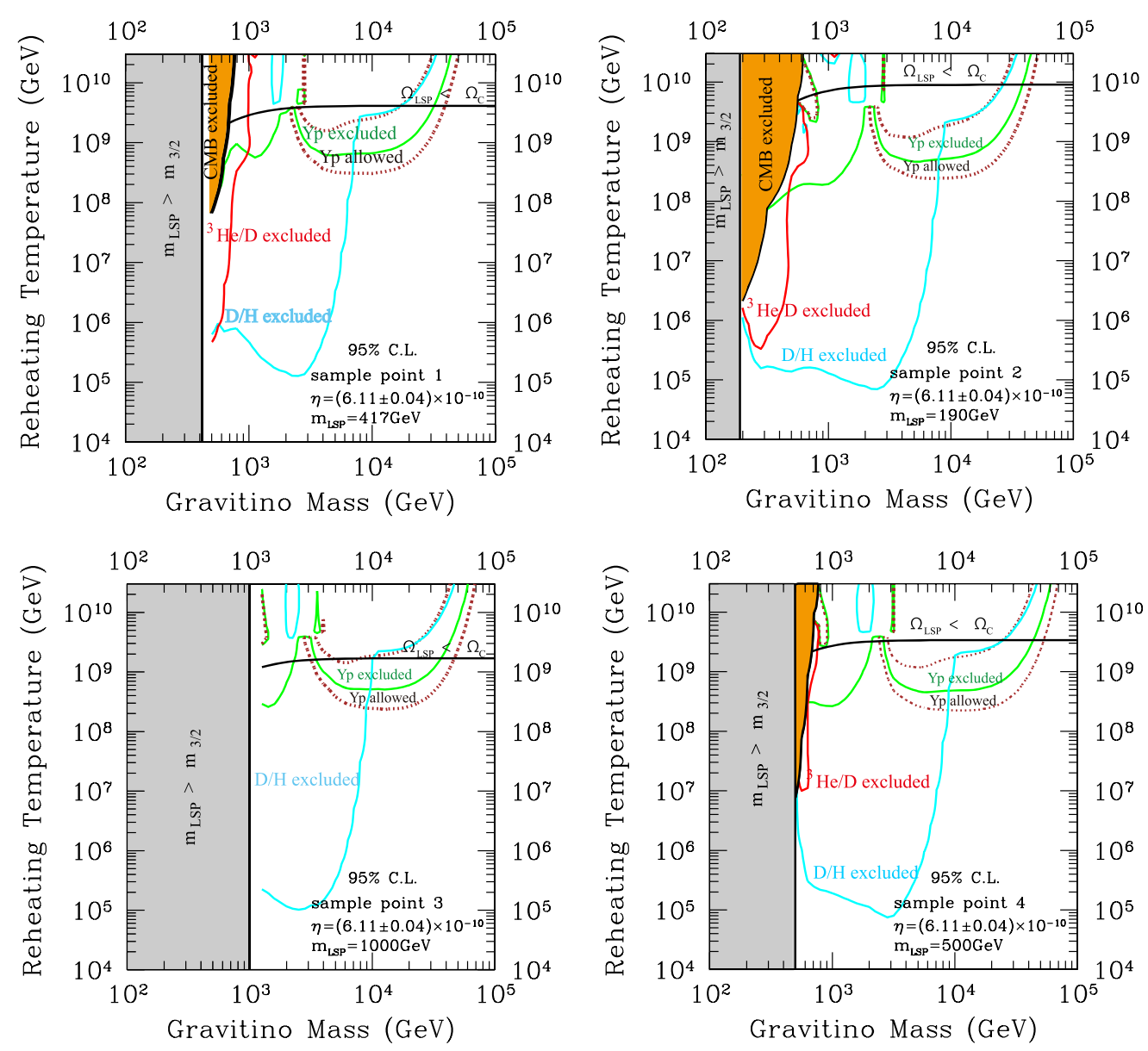

FIG. 16. Upper bound on the reheating temperature $T_{\mathrm{R}}$ as a function of $m_{3 / 2}$ at $95 \%$ C.L. for models of (1) point 1 (upper left panel), (2) point 2 (upper right panel), (3) point 3 (lower left panel), and (4) point 4 (lower right panel), respectively. The regions surrounded by the black-dotted line indicate a region consistent with Eq. (2.3).

the primordial ${ }^{4} \mathrm{He}$ abundance may become consistent with Eq. (2.3). In the present case, the gravitino has a sizable branching ratio for hadronic decay modes. In Fig. 16, we also show a region consistent with the ${ }^{4} \mathrm{He}$ abundance (2.3) estimated by Izotov et al. [38]. We can see that the allowed region exists for $m_{3 / 2} \sim \mathcal{O}(10) \mathrm{TeV}$ and $T_{\mathrm{R}} \sim \mathcal{O}\left(10^{9}\right) \mathrm{GeV}$.

Notice that the reheating temperature is bounded from above in order not to overclose the Universe by the LSP produced from the decay of the gravitino. For the parameter region of our interest, the gravitino decays at a cosmic temperature lower than the freeze-out temperature of the LSP. Thus, the density parameter of the LSP from the decay of the gravitino is evaluated as

$$
\Omega_{\mathrm{LSP}}^{\text {(decay) }}=\frac{m_{\mathrm{LSP}} Y_{3 / 2} s_{\text {now }}}{\rho_{\text {crit }}},
$$

where $s_{\text {now }}$ is the entropy density of the present Universe, and $\rho_{\text {crit }}$ is the critical density. We show the contour of $\Omega_{\mathrm{LSP}}^{\text {(decay) }}=\Omega_{c}$ (with $\Omega_{c} \simeq 0.26$ [41] being the density parameter of the cold dark matter). ${ }^{16}$ We can see that, with the present choice of the MSSM mass spectrum, the upper bound from the overclosure of the Universe is $10^{9}-10^{10} \mathrm{GeV}$, which is less stringent when the gravitino mass is smaller than $\sim 40-50 \mathrm{TeV}$.

Before closing this section, we comment on the implication of our result on leptogenesis [75], in which the baryon asymmetry of the Universe originates from the lepton asymmetry generated by the decay of righthanded neutrinos. In order to generate enough baryon asymmetry via thermal leptogenesis, the reheating temperature is required to be higher than $\sim 10^{9} \mathrm{GeV}[76,77]$. Thus, for a viable thermal leptogenesis scenario, we suggest scenarios realizing the gravitino mass of $\mathcal{O}(10) \mathrm{TeV}$, such as the pure gravity mediation scenario [65-67]. Notice that such a scenario works whether the

\footnotetext{
${ }^{16}$ If the thermal relic abundance of the LSP is sizable, the bound should be imposed on the total mass density of the LSP. However, because the thermal relic abundance is strongly dependent on the MSSM parameters, we show the bound based on $\Omega_{\mathrm{LSP}}^{\text {(decay) }}$.
} 
observational constraint on the ${ }^{4} \mathrm{He}$ abundance is Eq. (2.3) or (2.4).

\section{CONCLUSIONS AND DISCUSSION}

We have revisited and updated the BBN constraints on long-lived particles. Compared to the previous analysis, we have improved the following points. First, the SBBN reactions and their uncertainties have been updated. Second, we have revised the hadronic shower calculation by taking into account $p \leftrightarrow n$ conversion in the inelastic scattering of energetic nucleons off the background $p$ or ${ }^{4} \mathrm{He}$. Third, we have included the effects of the hadronic showers induced by the injections of energetic antinucleons $(\bar{p}$ and $\bar{n})$. Finally, we have used the most recent observational data for the abundance of ${ }^{4} \mathrm{He}$ and $\mathrm{D}$ and the cosmological parameters.

We have obtained the constraints on the abundance and lifetime of long-lived particles with various decay modes. They are shown in Figs. 12 and 13. The constraints become weaker when we include the $p \leftrightarrow n$ conversion effects in inelastic scattering because energetic neutrons change into protons and stop without causing hadrodissociation. On the other hand, inclusion of the energetic antinucleons makes the constraints more stringent. In addition, the recent precise measurement of the D abundance leads to stronger constraints. Thus, in total, the resultant constraints became more stringent than those obtained in the previous studies.

We have also applied our analysis to an unstable gravitino. We have adopted several patterns of the mass spectrum of superparticles and derived constraints on the reheating temperature after inflation, as shown in Fig. 16. The upper bound on the reheating temperature is $\sim 10^{5}-10^{6} \mathrm{GeV}$ for a gravitino mass $m_{3 / 2}$ less than several $\mathrm{TeV}$ and $\sim 10^{9} \mathrm{GeV}$ for $m_{3 / 2} \sim \mathcal{O}(10) \mathrm{TeV}$. This implies that the gravitino mass should be $\sim \mathcal{O}(10) \mathrm{TeV}$ for successful thermal leptogenesis.

In obtaining the constraints, we have adopted the observed ${ }^{4} \mathrm{He}$ abundance given by Eq. (2.4), which is consistent with SBBN. On the other hand, if we adopt the other estimation (2.3), ${ }^{4} \mathrm{He}$ abundance is inconsistent with SBBN. However, when long-lived particles with a large hadronic branch have a lifetime $\tau_{X} \sim 0.1-100 \mathrm{sec}$ and an abundance $m_{X} Y_{X} \sim 10^{-9}$, Eq. (2.3) becomes consistent with BBN.

In this work, we have not used ${ }^{7} \mathrm{Li}$ in deriving the constraints since the plateau value in ${ }^{7} \mathrm{Li}$ abundance observed in metal-poor stars (which had been considered as a primordial value) is smaller than the SBBN prediction by a factor of 2 to 3 (lithium problem) and, furthermore, the recent discovery of a much smaller ${ }^{7} \mathrm{Li}$ abundance in very metal-poor stars cannot be explained by any known mechanism. However, the effects of the decaying particles on the ${ }^{7} \mathrm{Li}$ and ${ }^{6} \mathrm{Li}$ abundance have been estimated in our numerical calculation. Interestingly, if we assume that the plateau value represents the primordial abundance, the decaying particles which mainly decay into $e^{+} e^{-}$ can solve the lithium problem for $\tau_{X} \sim 10^{2}-10^{3} \mathrm{sec}$ and $m_{X} Y_{X} \sim 10^{-7}$.

\section{ACKNOWLEDGMENTS}

We thank Benjamin Fuks and Olivier Mattelaer for their support in simulating gravitino decays with MadGraph5_aMC@NLO. We thank Jens Chluba for the useful discussions. This work was partially supported by JSPS KAKENHI Grants No. JP17H01131 (M. K. and K. K.), No. 17 K05434 (M. K.), No. 26247042 (K. K.), No. 16H06490 (T. M.), and No. 26400239 (T. M.), and MEXT KAKENHI Grants No. JP15H05889 (M. K. and K. K.), and No. JP16H0877 (K. K.).
[1] D. Lindley, Cosmological constraints on the lifetime of massive particles, Astrophys. J. 294, 1 (1985).

[2] M. Y. Khlopov and A. D. Linde, Is it easy to save the gravitino?, Phys. Lett. 138B, 265 (1984).

[3] F. Balestra et al., Sov. J. Nucl. Phys. 39, 626 (1984).

[4] J. R. Ellis, J. E. Kim, and D. V. Nanopoulos, Cosmological gravitino regeneration and decay, Phys. Lett. 145B, 181 (1984).

[5] R. Juszkiewicz, J. Silk, and A. Stebbins, Constraints on cosmologically regenerated gravitinos, Phys. Lett. 158B, 463 (1985).

[6] J. R. Ellis, D. V. Nanopoulos, and S. Sarkar, The cosmology of decaying gravitinos, Nucl. Phys. B259, 175 (1985).
[7] J. Audouze, D. Lindley, and J. Silk, Big bang photosynthesis and pregalactic nucleon synthesis of light elements, Astrophys. J. 293, L53 (1985).

[8] D. Lindley, Hadronic decays of cosmological gravitinos, Phys. Lett. B 171, 235 (1986).

[9] M. Kawasaki and K. Sato, Decay of gravitinos and photodestruction of light elements, Phys. Lett. B 189, 23 (1987).

[10] R. J. Scherrer and M. S. Turner, Primordial nucleosynthesis with decaying particles. I - Entropy-producing decays. II - Inert decays, Astrophys. J. 331, 19 (1988).

[11] J. R. Ellis, G. B. Gelmini, J. L. Lopez, D. V. Nanopoulos, and S. Sarkar, Astrophysical constraints on massive unstable neutral relic particles, Nucl. Phys. B373, 399 (1992). 
[12] M. Y. Khlopov, Y. L. Levitan, E. V. Sedelnikov, and I. M. Sobol, Nonequilibrium cosmological nucleosynthesis of light elements: Calculations by the Monte Carlo method, Yad. Fiz. 57, 1466 (1994) [Phys. At. Nucl. 57, 1393 (1994)].

[13] M. Kawasaki and T. Moroi, Gravitino production in the inflationary universe and the effects on big bang nucleosynthesis, Prog. Theor. Phys. 93, 879 (1995).

[14] M. Kawasaki and T. Moroi, Electromagnetic cascade in the early Universe and its application to the big bang nucleosynthesis, Astrophys. J. 452, 506 (1995).

[15] E. Holtmann, M. Kawasaki, K. Kohri, and T. Moroi, Radiative decay of a long-lived particle and big-bang nucleosynthesis, Phys. Rev. D 60, 023506 (1999).

[16] K. Jedamzik, Lithium-6: A Probe of the Early Universe, Phys. Rev. Lett. 84, 3248 (2000).

[17] M. Kawasaki, K. Kohri, and T. Moroi, Radiative decay of a massive particle and the nonthermal process in primordial nucleosynthesis, Phys. Rev. D 63, 103502 (2001).

[18] R. H. Cyburt, J. R. Ellis, B. D. Fields, and K. A. Olive, Updated nucleosynthesis constraints on unstable relic particles, Phys. Rev. D 67, 103521 (2003).

[19] M. Kusakabe, T. Kajino, T. Yoshida, T. Shima, Y. Nagai, and T. Kii, New constraints on radiative decay of long-lived $X$ particles in big bang nucleosynthesis with new rates of photodisintegration reactions of ${ }^{4} \mathrm{He}$, Phys. Rev. D 79, 123513 (2009).

[20] V. Poulin and P. D. Serpico, Nonuniversal BBN bounds on electromagnetically decaying particles, Phys. Rev. D 91, 103007 (2015).

[21] M. H. Reno and D. Seckel, Primordial nucleosynthesis: The effects of injecting hadrons, Phys. Rev. D 37, 3441 (1988).

[22] S. Dimopoulos, R. Esmailzadeh, L. J. Hall, and G. D. Starkman, Is the Universe closed by baryons? Nucleosynthesis with a late decaying massive particle, Astrophys. J. 330, 545 (1988).

[23] S. Dimopoulos, R. Esmailzadeh, L. J. Hall, and G. D. Starkman, Kiloelectronvolt-Era Nucleosynthesis and Its Implications, Phys. Rev. Lett. 60, 7 (1988).

[24] S. Dimopoulos, R. Esmailzadeh, L. J. Hall, and G. D. Starkman, Limits on late decaying particles from nucleosynthesis, Nucl. Phys. B311, 699 (1989).

[25] K. Kohri, Primordial nucleosynthesis and hadronic decay of a massive particle with a relatively short lifetime, Phys. Rev. D 64, 043515 (2001).

[26] K. Jedamzik, Did something decay, evaporate, or annihilate during big bang nucleosynthesis?, Phys. Rev. D 70, 063524 (2004).

[27] M. Kawasaki, K. Kohri, and T. Moroi, Hadronic decay of late-decaying particles and big-bang nucleosynthesis, Phys. Lett. B 625, 7 (2005).

[28] M. Kawasaki, K. Kohri, and T. Moroi, Big-bang nucleosynthesis and hadronic decay of long-lived massive particles, Phys. Rev. D 71, 083502 (2005).

[29] K. Kohri, T. Moroi, and A. Yotsuyanagi, Big-bang nucleosynthesis with unstable gravitino and upper bound on the reheating temperature, Phys. Rev. D 73, 123511 (2006).

[30] K. Jedamzik, Big bang nucleosynthesis constraints on hadronically and electromagnetically decaying relic neutral particles, Phys. Rev. D 74, 103509 (2006).
[31] M. Kawasaki, K. Kohri, T. Moroi, and A. Yotsuyanagi, Big-bang nucleosynthesis and gravitino, Phys. Rev. D 78, 065011 (2008).

[32] R. H. Cyburt, J. Ellis, B. D. Fields, F. Luo, K. A. Olive, and V.C. Spanos, Nucleosynthesis constraints on a massive gravitino in neutralino dark matter scenarios, J. Cosmol. Astropart. Phys. 10 (2009) 021.

[33] S. Weinberg, Cosmological Constraints on the Scale of Supersymmetry Breaking, Phys. Rev. Lett. 48, 1303 (1982).

[34] M. Kawasaki, K. Kohri, T. Moroi, and Y. Takaesu, Revisiting big-bang nucleosynthesis constraints on dark-matter annihilation, Phys. Lett. B 751, 246 (2015).

[35] R. Cooke, M. Pettini, R. A. Jorgenson, M. T. Murphy, and C. C. Steidel, Precision measures of the primordial abundance of deuterium, Astrophys. J. 781, 31 (2014).

[36] G. Sigl, K. Jedamzik, D. N. Schramm, and V. S. Berezinsky, Helium photodisintegration and nucleosynthesis: Implications for topological defects, high-energy cosmic rays, and massive black holes, Phys. Rev. D 52, 6682 (1995).

[37] J. Geiss and G. Gloeckler, Isotopic composition of H, HE and NE in the protosolar cloud, Space Sci. Rev. 106, 3 (2003).

[38] Y. I. Izotov, T.X. Thuan, and N. G. Guseva, A new determination of the primordial He abundance using the HeI 10830A emission line: Cosmological implications, Mon. Not. R. Astron. Soc. 445, 778 (2014).

[39] E. Aver, K. A. Olive, and E. D. Skillman, The effects of He I $\lambda 10830$ on helium abundance determinations, J. Cosmol. Astropart. Phys. 07 (2015) 011.

[40] L. Sbordone, P. Bonifacio, E. Caffau, H.-G. Ludwig, N. T. Behara, J. I. G. Hernandez, M. Steffen, R. Cayrel et al., The metal-poor end of the Spite plateau. I. Stellar parameters, metallicities and lithium abundances, Astron. Astrophys. 522, A26 (2010).

[41] P. A. R. Ade et al. (Planck Collaboration), Planck 2015 results. XIII. Cosmological parameters, Astron. Astrophys. 594, A13 (2016).

[42] T. Sjöstrand, S. Ask, J. R. Christiansen, R. Corke, N. Desai, P. Ilten, S. Mrenna, S. Prestel, C. O. Rasmussen, and P.Z. Skands, An introduction to PYTHIA8.2, Comput. Phys. Commun. 191, 159 (2015).

[43] P. D. Serpico, S. Esposito, F. Iocco, G. Mangano, G. Miele, and O. Pisanti, Nuclear reaction network for primordial nucleosynthesis: A detailed analysis of rates, uncertainties and light nuclei yields, J. Cosmol. Astropart. Phys. 12 (2004) 010.

[44] R.H. Cyburt and B. Davids, Evaluation of modern ${ }^{3} \mathrm{He}(\alpha, \gamma){ }^{7} \mathrm{Be}$ data, Phys. Rev. C 78, 064614 (2008).

[45] J. P. Meyer, Astron. Astrophys. Suppl. Ser. 7, 417 (1972).

[46] C. Patrignani et al. (Particle Data Group), Review of particle physics, Chin. Phys. C 40, 100001 (2016).

[47] E. Bracci, J. P. Droulez, E. Flaminio, J. D. Hansen, D. R. O. Morrison, and Douglas Robert Ogston, CERN Report No. CERN-HERA-73-1, 1973.

[48] J. Hisano, M. Kawasaki, K. Kohri, T. Moroi, and K. Nakayama, Cosmic rays from dark matter annihilation and big-bang nucleosynthesis, Phys. Rev. D 79, 083522 (2009).

[49] R. J. Scherrer, J. M. Cline, S. Raby, and D. Seckel, Gravitino induced baryogenesis, primordial nucleosynthesis, and the Tremaine-Gunn limit, Phys. Rev. D 44, 3760 (1991). 
[50] W. Hu and J. Silk, Thermalization Constraints and Spectral Distortions for Massive Unstable Relic Particles, Phys. Rev. Lett. 70, 2661 (1993).

[51] J. Chluba and R. A. Sunyaev, The evolution of CMB spectral distortions in the early Universe, Mon. Not. R. Astron. Soc. 419, 1294 (2012).

[52] J. Chluba, Distinguishing different scenarios of early energy release with spectral distortions of the cosmic microwave background, Mon. Not. R. Astron. Soc. 436, 2232 (2013).

[53] D. J. Fixsen, E. S. Cheng, J. M. Gales, J. C. Mather, R. A. Shafer, and E. L. Wright, The cosmic microwave background spectrum from the full COBE FIRAS data set, Astrophys. J. 473, 576 (1996).

[54] E. Dimastrogiovanni, L. M. Krauss, and J. Chluba, Constraints on gravitino decay and the scale of inflation using CMB spectral distortions, Phys. Rev. D 94, 023518 (2016).

[55] J. Chluba and D. Jeong, Teasing bits of information out of the CMB energy spectrum, Mon. Not. R. Astron. Soc. 438, 2065 (2014).

[56] K. Ishiwata, M. Kawasaki, K. Kohri, and T. Moroi, Righthanded sneutrino dark matter and big-bang nucleosynthesis, Phys. Lett. B 689, 163 (2010).

[57] M. Endo, K. Hamaguchi, and F. Takahashi, Moduli-Induced Gravitino Problem, Phys. Rev. Lett. 96, 211301 (2006).

[58] S. Nakamura and M. Yamaguchi, Gravitino production from heavy moduli decay and cosmological moduli problem revived, Phys. Lett. B 638, 389 (2006).

[59] M. Kawasaki, F. Takahashi, and T. T. Yanagida, Gravitino overproduction in inflaton decay, Phys. Lett. B 638, 8 (2006).

[60] M. Bolz, A. Brandenburg, and W. Buchmuller, Thermal production of gravitinos, Nucl. Phys. B606, 518 (2001); Erratum, Nucl. Phys. 790B, 336 (2008).

[61] J. Pradler and F. D. Steffen, Thermal gravitino production and collider tests of leptogenesis, Phys. Rev. D 75, 023509 (2007).

[62] V.S. Rychkov and A. Strumia, Thermal production of gravitinos, Phys. Rev. D 75, 075011 (2007).

[63] J. Ellis, M. A. G. Garcia, D. V. Nanopoulos, K. A. Olive, and M. Peloso, Post-inflationary gravitino production revisited, J. Cosmol. Astropart. Phys. 03 (2016) 008.

[64] S. P. Martin, in Perspectives on Supersymmetry II, edited by G. L. Kane, Advanced Series on Directions in High Energy Physics Vol. 21 (World Scientific, Singapore, 2010), p. 1; in Perpectives on Supersymmetry, edited by G. L. Kane,
Advanced Series on Directions in High Energy Physics Vol. 18 (World Scientific, Singapore, 1998), p. 1.

[65] M. Ibe, T. Moroi, and T. T. Yanagida, Possible signals of wino LSP at the Large Hadron Collider, Phys. Lett. B 644, 355 (2007).

[66] M. Ibe and T. T. Yanagida, The lightest Higgs boson mass in pure gravity mediation model, Phys. Lett. B 709, 374 (2012).

[67] N. Arkani-Hamed, A. Gupta, D. E. Kaplan, N. Weiner, and T. Zorawski, Simply unnatural supersymmetry, arXiv: 1212.6971.

[68] L. Randall and R. Sundrum, Out of this world supersymmetry breaking, Nucl. Phys. B557, 79 (1999).

[69] G. F. Giudice, M. A. Luty, H. Murayama, and R. Rattazzi, Gaugino mass without singlets, J. High Energy Phys. 12 (1998) 027.

[70] J. Alwall, R. Frederix, S. Frixione, V. Hirschi, F. Maltoni, O. Mattelaer, H.-S. Shao, T. Stelzer, P. Torrielli, and M. Zaro, The automated computation of tree-level and next-toleading order differential cross sections, and their matching to parton shower simulations, J. High Energy Phys. 07 (2014) 079.

[71] C. Duhr and B. Fuks, A superspace module for the FeynRules package, Comput. Phys. Commun. 182, 2404 (2011).

[72] C. Degrande, C. Duhr, B. Fuks, D. Grellscheid, O. Mattelaer, and T. Reiter, UFO-The universal FeynRules output, Comput. Phys. Commun. 183, 1201 (2012).

[73] N. D. Christensen, P. de Aquino, N. Deutschmann, C. Duhr, B. Fuks, C. Garcia-Cely, O. Mattelaer, K. Mawatari, B. Oexl, and Y. Takaesu, Simulating spin- $\frac{3}{2}$ particles at colliders, Eur. Phys. J. C 73, 2580 (2013).

[74] A. Alloul, N. D. Christensen, C. Degrande, C. Duhr, and B. Fuks, FeynRules 2.0-A complete toolbox for tree-level phenomenology, Comput. Phys. Commun. 185, 2250 (2014).

[75] M. Fukugita and T. Yanagida, Baryogenesis without grand unification, Phys. Lett. B 174, 45 (1986).

[76] G. F. Giudice, A. Notari, M. Raidal, A. Riotto, and A. Strumia, Towards a complete theory of thermal leptogenesis in the SM and MSSM, Nucl. Phys. B685, 89 (2004).

[77] W. Buchmuller, P. Di Bari, and M. Plumacher, Leptogenesis for pedestrians, Ann. Phys. (Amsterdam) 315, 305 (2005).

Correction: The previously published Figures 11-16 contained minor inaccuracies and have been fixed. 